KUL-TF-04/21

hep-th/0407233

\title{
O3/O7 Orientifold Truncations and Very Special Quaternionic-Kähler Geometry
}

\author{
Geert Smet and Joris Van den Bergh ${ }^{\dagger}$ \\ Instituut voor Theoretische Fysica, Katholieke Universiteit Leuven, \\ Celestijnenlaan 200D B-3001 Leuven, Belgium.
}

\begin{abstract}
We study the orientifold truncation that arises when compactifying type II string theory on Calabi-Yau orientifolds with O3/O7-planes, in the context of supergravity. We look at the $\mathrm{N}=2$ to $\mathrm{N}=1$ reduction of the hypermultiplet sector of $\mathrm{N}=2$ supergravity under the truncation, for the case of very special quaternionic-Kähler target space geometry. We explicitly verify the Kähler structure of the truncated spaces, and we study the truncated isometry algebra. For symmetric special quaternionic spaces, we give a complete overview of the spaces one finds after truncation. We also find new examples of 'dual' Kähler spaces, that give rise to flat potentials in $\mathrm{N}=1$ supergravity.
\end{abstract}

$\mp\{$ geert.smet, joris.vandenbergh\}@fys.kuleuven.ac.be 


\section{Introduction}

In this paper, we study the $N=2 \rightarrow N=1$ reduction of the hypermultiplet sector of $N=2$ supergravity that one gets by performing an orientifold truncation with $03 / O 7$ planes. This truncation was discussed by Grana et al. in 1] and further studied by Grimm and Louis in [2]. There, the authors describe the $N=1$ low energy effective action for compactifications of type IIB string theory on Calabi-Yau orientifolds in the presence of background fluxes.

We will study the orientifold truncation in the context of supergravity, with the aim of describing the effect of the truncation on the class of very special quaternionic-Kähler hypermultiplet moduli spaces. These spaces arise naturally when one looks at compactifications of type IIA/IIB string theory on Calabi-Yau spaces. For the IIA case, it is known that the low energy spectrum contains the universal hypermultiplet (containing the dilaton, the dual of an anti-symmetric tensor, and two scalars from the R-R sector) and $n=h_{(1,2)}$ additional hypermultiplets. For the IIB case, one also finds $n+1$ hypermultiplets in the low energy spectrum, after dualizing the double-tensor multiplet and $n=h_{(1,1)}$ additional tensor multiplets. The tree level lagrangian for the $n+1$ hypermultiplets was calculated in 3]. The scalar manifold spanned by the $4 n+4$ scalars is constrained by $N=2$ supergravity to be a quaternionic-Kähler manifold [4], as was explicitly verified in 3. Furthermore, the scalar manifold is constrained by the c-map to be of special quaternionic type: the geometry is completely determined by a holomorphic prepotential $F(X)$, which is homogeneous of degree two. The very special structure arises when one takes the large-volume limit for IIB compactifications or the 'large complex structure limit' for the IIA case (for a review, see [5]): the prepotential in this limit

is given by $F=i d_{A B C} \frac{X^{A} X^{B} X^{C}}{X^{0}}$ with $d_{A B C}$ a completely symmetric tensor. Taking these limits is necessary to display the mirror symmetry map between IIA and IIB coordinates, as discussed in [6]. In this paper, we will always use IIA coordinates.

In the supergravity context, very special quaternionic spaces arise when performing dimensional reduction from $D=5$ to $D=3$ by applying the $\mathbf{r}$-map followed by the $\mathbf{c}$-map. These maps give the following relation between very special real, Kähler and quaternionicKähler spaces:

$$
\mathbb{R}_{n-1} \stackrel{\mathbf{r}}{\longrightarrow} \mathbb{C}_{n} \stackrel{\mathbf{c}}{\longrightarrow} \mathbb{H}_{n+1},
$$

where $n-1, n$ and $n+1$ denote the real, complex and quaternionic dimensions respectively (see [7] for an exhaustive review).

Compactification on a Calabi-Yau orientifold results in the $N=2 \rightarrow N=1$ breaking of supersymmetry. The effect of the orientifold truncation is to put to zero half of the scalars of the quaternionic-Kähler manifold $\mathbb{H}_{n+1}$, so that the truncated manifold has real dimension $2 n+2$. Furthermore, it has to be Kähler, due to the remaining $N=1$ supersymmetry.

The case corresponding to the presence of only $O 3$-planes was already studied in detail by D'Auria et. al. in [8] and [9] (see als [10, where stringy corrections were discussed). They found that the truncated manifold factorizes into a $2 n$-dimensional Kähler space times the space $S U(1,1) / U(1)$. The $2 n$-dimensional space can be viewed as 'dual' to the 
original $2 n$-dimensional Kähler space (denoted $\mathbb{C}_{n}$ in (1.1)), that is mapped by the c-map into the quaternionic space.

We will study the truncation of very special quaternionic-Kähler manifolds that corresponds to the presence of both $\mathrm{O} 3$ - and $\mathrm{O} 7$-planes. In section 2 we introduce the orientifold truncation. In section 3 we show that the truncated quaternionic-Kähler manifolds are Kähler, by explicitly computing the Kähler potential. In the following sections we analyze the isometry algebra of the truncated manifolds: we study in detail the case of homogeneous quaternionic spaces, and for the case of symmetric spaces, we give a complete overview of which spaces one finds after truncation. We also extend the classification to include the symmetric special quaternionic spaces that are not very special. Finally, in the last section we look at the curvature tensor of the truncated manifolds and we find a new class of Kähler potentials that lead to flat potentials in $N=1$ supergravity.

\section{The orientifold truncation}

First we fix our notation: we will take the same notation as the one used by D'Auria et al. in [8], except that we rename some of the scalars to conform to the the notation used by de Wit et. al. in [7, to which we shall refer extensively. The $(4 n+4)$-dimensional very special quaternionic manifolds are parametrized by:

- the special coordinates $z^{A}=\frac{1}{2}\left(x^{A}+i y^{A}\right), \quad A=1, \ldots, n$,

- the extra coordinates $D, \sigma, A^{I}, B_{I}, \quad I=1, \ldots, n+1$.

The identification with [8] is provided by

$$
\begin{aligned}
& x^{A} \rightarrow x^{A}, \quad y^{A} \rightarrow y^{A}, \\
& A^{I} \rightarrow \zeta^{I}, \quad B_{I} \rightarrow \tilde{\zeta}_{I}, \\
& D \rightarrow D, \quad \sigma \rightarrow \tilde{\Phi} .
\end{aligned}
$$

The O3/O7-orientifold truncation is described in [8], [1]: the set of special coordinates $z^{A}$ is separated in two parts with opposite chirality $z_{ \pm}^{A}\left(n_{+}+n_{-}=n\right)$ such that:

$$
\begin{aligned}
& y_{ \pm} \rightarrow \pm y_{ \pm}, \\
& x_{ \pm} \rightarrow \mp x_{ \pm},
\end{aligned}
$$

and

$$
\begin{aligned}
& A_{ \pm} \rightarrow \pm A_{\mp}, \quad A^{0} \rightarrow A^{0}, \\
& B_{ \pm} \rightarrow \pm B_{ \pm}, \quad B_{0} \rightarrow-B_{0}, \\
& D \rightarrow D, \quad \sigma \rightarrow-\sigma .
\end{aligned}
$$

The case $n_{-}=0$ (only O3-planes present) was studied in detail in [8, 9]. We will extend these results to the case $n_{-} \neq 0$. We split the index $A$ into $A=(a, \alpha)$, with $a=1, . ., n_{-}$ 
and $\alpha=1, . ., n_{+}$. We can now restrict to the plus-parity sector by performing the following truncation

$$
\begin{aligned}
& x^{\alpha}=y^{a}=\sigma=0, \\
& A^{\alpha}=B_{a}=B_{0}=0 .
\end{aligned}
$$

The remaining non-zero fields are: $\left\{D, x^{a}, y^{\alpha}, A^{0}, A^{a}, B_{\alpha}\right\}$.

For a consistent truncation, one must also demand [1], 12,

$$
d_{\alpha \beta a}=d_{a b c}=0 .
$$

For later use, we introduce the following notation *:

$$
\begin{aligned}
& (\kappa y)_{\alpha \beta}=d_{\alpha \beta \gamma} y^{\gamma},(\kappa y)_{a b}=d_{a b \gamma} y^{\gamma},(\kappa x)_{\alpha a}=d_{a b \alpha} x^{b} \\
& (\kappa y y)_{\alpha}=d_{\alpha \beta \gamma} y^{\beta} y^{\gamma},(\kappa x y)_{a}=d_{a b \gamma} x^{b} y^{\gamma},(\kappa x x)_{\alpha}=d_{a b \alpha} x^{a} x^{b} \\
& (\kappa y y y)=d_{\alpha \beta \gamma} y^{\alpha} y^{\beta} y^{\gamma}=\kappa,(\kappa x x y)=d_{a b \gamma} x^{a} x^{b} y^{\gamma}, V(y)=\frac{1}{6}(\kappa y y y)
\end{aligned}
$$

The $N=2$ lagrangian for $n+1$ hypermultiplets with very special quaternionic-Kähler target space is (see [6]):

$$
\begin{aligned}
\mathcal{L}= & -\frac{1}{2} R-\left(\partial_{\mu} D\right)^{2}-\frac{1}{4} G_{A B} \partial_{\mu} y^{A} \partial^{\mu} y^{B}-\frac{1}{4} G_{A B} \partial_{\mu} x^{A} \partial^{\mu} x^{B} \\
- & -\frac{1}{8} e^{2 D} V\left(\partial_{\mu} A^{0}\right)^{2}-\frac{1}{2} e^{2 D} V G_{A B}\left(x^{B} \partial_{\mu} A^{0}-\partial_{\mu} A^{B}\right)\left(x^{A} \partial^{\mu} A^{0}-\partial^{\mu} A^{A}\right) \\
- & 2 e^{2 D} V^{-1} G^{-1 A B}\left(\partial_{\mu} B_{A}+\frac{1}{8}(\kappa x x)_{A} \partial_{\mu} A^{0}-\frac{1}{4}(\kappa x)_{A C} \partial_{\mu} A^{C}\right) \times \\
& \left(\partial^{\mu} B_{B}+\frac{1}{8}(\kappa x x)_{B} \partial^{\mu} A^{0}-\frac{1}{4}(\kappa x)_{B D} \partial^{\mu} A^{D}\right) \\
- & 2 e^{2 D} V^{-1}\left(\partial_{\mu} B_{0}+x^{A} \partial_{\mu} B_{A}+\frac{1}{24}(\kappa x x x) \partial_{\mu} A^{0}-\frac{1}{8}(\kappa x x)_{A} \partial_{\mu} A^{A}\right)^{2} \\
- & \frac{1}{4} e^{4 D}\left(\partial_{\mu} \sigma+A^{C} \partial_{\mu} B_{C}-B^{C} \partial_{\mu} A_{C}\right)^{2},
\end{aligned}
$$

with

$$
G_{A B}=-6\left(\frac{(\kappa y)_{A B}}{(\kappa y y y)}-\frac{3}{2} \frac{(\kappa y y)_{A}(\kappa y y)_{B}}{(d y y y)^{2}}\right)
$$

Performing the truncation (2.3) now gives rise to the following lagrangian with $2 n+2$ real variables (we omit the trivially contracted index $\mu$ from here on):

$$
\begin{aligned}
\sqrt{-g}^{-1} \mathcal{L}= & -(\partial D)^{2}-\frac{1}{4} G_{\alpha \beta} \partial y^{\alpha} \partial y^{\beta}-\frac{1}{4} G_{a b} \partial x^{a} \partial x^{b}-\frac{1}{8} e^{2 D} V\left(\partial A^{0}\right)^{2} \\
& -\frac{1}{8} e^{2 D} V G_{a b}\left(x^{a} \partial A^{0}-\partial A^{a}\right)\left(x^{b} \partial A^{0}-\partial A^{b}\right)
\end{aligned}
$$

*It will always be clear which variables have a greek index, and which have a latin index, so that there should not be any confusion between $(\kappa x x)_{\alpha}$ and $(\kappa y y)_{\alpha}$. 


$$
\begin{aligned}
& -2 e^{2 D} V^{-1}\left(G^{-1}\right)^{\alpha \beta}\left[\partial B_{\alpha}+\frac{1}{8}(\kappa x x)_{\alpha} \partial A^{0}\right. \\
& \left.-\frac{1}{4}(\kappa x)_{\alpha c} \partial A^{c}\right]\left[\partial B_{\beta}+\frac{1}{8}(\kappa x x)_{\beta} \partial A^{0}-\frac{1}{4}(\kappa x)_{\beta d} \partial A^{d}\right],
\end{aligned}
$$

with truncated elements of the metric given by

$$
\begin{aligned}
G_{\alpha \beta} & =-6\left(\frac{(\kappa y)_{\alpha \beta}}{(\kappa y y y)}-\frac{3}{2} \frac{(\kappa y y)_{\alpha}(\kappa y y)_{\beta}}{(d y y y)^{2}}\right), \\
G_{\alpha a} & =0, \\
G_{a b} & =-6 \frac{(\kappa y)_{a b}}{(\kappa y y y)} .
\end{aligned}
$$

Note that the metric has no mixed components. From this, and the restriction (2.4) on the $d$-symbols, one can deduce a restriction on the matrix $C^{A B C}$ that appears in the curvature tensor (see [7]). It is defined by

$$
C^{A B C}=-27 G^{A D} G^{B E} G^{C F} d_{D E F}(\kappa y y y)^{-2},
$$

and one sees easily that

$$
C^{\alpha \beta a}=C^{a b c}=0
$$

\section{Calculation of the Kähler Potential}

The $N=1 \sigma$-model Lagrangian resulting from the orientifolding is given by (2.8). Supersymmetry requires the target space to be Kähler, so the aim is to find the right coordinate transformations that allow us to write this lagrangian in complex coordinates and to find the Kähler potential. We start with the same change of variables as in [8]

$$
e^{2 \Phi}:=V(y) e^{2 D}, \quad \chi^{\alpha}:=y^{\alpha} e^{-\frac{\Phi}{2}}
$$

followed by

$$
p_{\alpha}:=\frac{1}{2}(\kappa \chi \chi)_{\alpha}=\frac{1}{2} d_{\alpha \beta \gamma} \chi^{\beta} \chi^{\gamma}
$$

so that

$$
\partial \chi^{\beta}=\left((\kappa \chi)^{-1}\right)^{\beta \alpha} \partial p_{\alpha}
$$

Now we define

$$
\lambda^{a}:=x^{a} e^{-\Phi},
$$

and we also perform a rescaling $A^{a} \rightarrow A^{a} \sqrt{2}, A^{0} \rightarrow A^{0} \sqrt{2}$ and $B_{\alpha} \rightarrow B_{\alpha} / 2 \sqrt{2}$.

This gives us the following lagrangian in the new variables:

$$
\begin{aligned}
(\sqrt{-g})^{-1} \mathcal{L}= & -\frac{1}{4} e^{\Phi} G_{a b}(\chi)\left[\partial \lambda^{a} \partial \lambda^{b}+\partial A^{a} \partial A^{b}\right] \\
& +\frac{1}{2} e^{\Phi} G_{a b} \lambda^{b}\left[-\partial \Phi \partial \lambda^{a}+e^{\Phi} \partial A^{a} \partial A^{0}\right]
\end{aligned}
$$




$$
\begin{aligned}
& -\frac{1}{4}\left(G_{a b}+1\right) \lambda^{a} \lambda^{b}\left[(\partial \Phi)^{2}+e^{2 \Phi}\left(\partial A^{0}\right)^{2}\right] \\
& -\frac{1}{4} V(\lambda)^{-2}\left(G^{-1}\right)^{\alpha \beta}\left[\partial B_{\alpha} \partial B_{\beta}+\partial p_{\alpha} \partial p_{\beta}+e^{2 \Phi}(\kappa \lambda \lambda)_{\beta} \partial A^{0} \partial B_{\alpha}\right. \\
& -2 e^{\Phi}(\kappa \lambda)_{\beta c} \partial B_{\alpha} \partial A^{c}+\frac{1}{4} e^{4 \Phi}(\kappa \lambda \lambda)_{\alpha}(\kappa \lambda)_{\beta}\left(\partial A^{0}\right)^{2} \\
& \left.e^{3 \Phi}(\kappa \lambda \lambda)_{\alpha}(\kappa \lambda)_{\beta c} \partial A^{0} \partial A^{c}+e^{2 \Phi}(\kappa \lambda)_{\alpha c}(\kappa \lambda)_{\beta d} \partial A^{c} \partial A^{d}\right] .
\end{aligned}
$$

Finally, we perform the following transformation:

$$
p_{\alpha}=-t_{\alpha}+\frac{1}{2} e^{\Phi}(\kappa \lambda \lambda)_{\alpha}=-t_{\alpha}+\frac{1}{2} e^{\Phi} d_{\alpha a b} \lambda^{a} \lambda^{b} .
$$

After some calculation, we find that the lagrangian can now be written in the following complex coordinates:

$$
\begin{aligned}
& \eta=\exp (-\Phi)+i A^{o}=\eta_{1}+i \eta_{2}, \\
& \epsilon_{\alpha}=t_{\alpha}+i B_{\alpha}, \\
& \rho^{a}=\lambda^{a}+i A^{a} .
\end{aligned}
$$

This gives us

$$
\begin{aligned}
\sqrt{-g^{-1}} \mathcal{L}= & -\frac{1}{4}\left[\frac{G_{a b}}{\eta_{1}}+\frac{g^{\alpha \beta}}{\eta_{1}{ }^{2}}(\kappa \lambda)_{\alpha a}(\kappa \lambda)_{\beta b}\right]\left[\partial \rho^{a} \partial \bar{\rho}^{b}\right]-\frac{1}{4} g^{\alpha \beta}\left[\partial \epsilon_{\alpha} \partial \bar{\epsilon}_{\beta}\right] \\
& -\frac{1}{4 \eta_{1}{ }^{2}}\left[1+\frac{1}{4 \eta_{1}{ }^{2}} g^{\alpha \beta}(\kappa \lambda \lambda)_{\alpha}(\kappa \lambda \lambda)_{\beta}+\frac{1}{\eta} G_{a b} \lambda^{a} \lambda^{b}\right](\partial \eta)^{2} \\
& +\frac{1}{4 \eta_{1}{ }^{2}}\left[G_{a b} \lambda^{b}+\frac{1}{2 \eta_{1}} g^{\alpha \beta}(\kappa \lambda \lambda)_{\alpha}(\kappa \lambda)_{\beta a}\right]\left[\partial \eta \partial \bar{\rho}^{a}+\partial \bar{\eta} \partial \rho^{a}\right] \\
& +\frac{1}{4 \eta_{1}} g^{\alpha \beta}(\kappa \lambda)_{\beta a}\left[\partial \epsilon_{\alpha} \partial \bar{\rho}^{a}+\partial \bar{\epsilon}_{\alpha} \partial \rho^{a}\right] \\
& -\frac{1}{8 \eta_{1}{ }^{2}} g^{\alpha \beta}(\kappa \lambda \lambda)_{\beta}\left[\partial \epsilon_{\alpha} \partial \bar{\eta}+\partial \eta \partial \bar{\epsilon}_{\alpha}\right],
\end{aligned}
$$

with $g^{\alpha \beta}:=\left(G^{-1}\right)^{\alpha \beta} / V^{2}$.

The metric can be derived from the following Kähler-potential:

$$
K=-2 \log V\left(\chi^{\alpha}\left(p\left(t_{\beta}, \lambda^{a}, \eta_{1}\right)\right)\right)-\log \left(\eta_{1}\right),
$$

since we can write the Lagrangian as

$$
\begin{aligned}
\sqrt{-g^{-1}} \mathcal{L}= & -\frac{1}{4}\left[\bar{g}_{a b}\left(\partial \rho^{a} \partial \bar{\rho}^{b}\right)+\bar{g}^{\alpha \beta}\left(\partial \epsilon_{\alpha} \partial \bar{\epsilon}_{\beta}\right)+\bar{g}_{a 0}\left(\partial \eta \partial \bar{\rho}^{a}+\partial \bar{\eta} \partial \rho^{a}\right)\right. \\
& \left.+\bar{g}_{00}(\partial \eta)^{2}+\bar{g}^{\alpha}{ }_{a}\left(\partial \epsilon_{\alpha} \partial \bar{\rho}^{a}+\partial \bar{\epsilon}_{\alpha} \partial \rho^{a}\right)+\bar{g}^{\alpha}{ }_{0}\left(\partial \epsilon_{\alpha} \partial \bar{\eta}+\partial \eta \partial \bar{\epsilon}_{\alpha}\right)\right],
\end{aligned}
$$

where

$$
\begin{aligned}
\bar{g}^{\alpha \beta}=\frac{\partial^{2} K}{\partial t_{\alpha} \partial t_{\beta}}, & \bar{g}_{00}=\frac{\partial^{2} K}{\partial \eta_{1} \partial \eta_{1}}, & \bar{g}_{a b}=\frac{\partial^{2} K}{\partial \lambda^{a} \partial \lambda^{b}}, \\
\bar{g}_{a 0}=\frac{\partial^{2} K}{\partial \lambda^{a} \partial \eta_{1}}, & \bar{g}^{\alpha}=\frac{\partial^{2} K}{\partial t_{\alpha} \partial \eta_{1}}, & \bar{g}_{a}^{\alpha}=\frac{\partial^{2} K}{\partial t_{\alpha} \partial \lambda^{a}} .
\end{aligned}
$$


Thus, one obtains the potential by starting with the Kähler potential of [8] and applying to it the transformation (3.6). The Kähler potential depends only on the real parts of the complex variables. Contrary to the case $n_{-}=0$ studied in [8], the $(\eta, \bar{\eta})$-part does not decouple when $n_{-} \neq 0$, and therefore does not describe a seperate $S U(1,1) / U(1) \sigma$-model. The Kähler space that is obtained, has complex dimension $n+1$ and is therefore certainly distinct from the original Kähler space upon which the c-map is performed (which has complex dimension $n$ ).

It is also interesting to see whether the resulting $N=1$ target space can give rise to no-scale models ([13, 14, 15]). To study this, we need the inverse metric. Defining $\left(\eta^{\Lambda}\right)=\left(\eta, \epsilon_{\alpha}, \rho^{a}\right)$, we have for the metric $\bar{g}_{\Lambda \bar{\Sigma}}$

$$
\begin{aligned}
\bar{g}^{\alpha \beta} & =g^{\alpha \beta} \\
\bar{g}_{00} & =\frac{1}{\eta_{1}^{2}}\left(1+\frac{1}{4 \eta_{1}^{2}} g^{\alpha \beta}(\kappa \lambda \lambda)_{\alpha}(\kappa \lambda \lambda)_{\beta}+\frac{1}{\eta_{1}} G_{a b} \lambda^{a} \lambda^{b}\right), \\
\bar{g}_{a b} & =\frac{g^{\alpha \beta}}{\eta_{1}^{2}}(\kappa \lambda)_{\alpha a}(\kappa \lambda)_{\beta b}+\frac{1}{\eta_{1}} G_{a b}, \\
\bar{g}_{a 0} & =-\left(\frac{1}{\eta_{1}^{2}} G_{a b} \lambda^{b}+\frac{1}{2 \eta_{1}^{3}} g^{\alpha \beta}(\kappa \lambda \lambda)_{\beta}(\kappa \lambda)_{\alpha a}\right), \\
\bar{g}_{0}^{\alpha} & =\frac{1}{2 \eta_{1}^{2}} g^{\alpha \beta}(\kappa \lambda \lambda)_{\beta}, \\
\bar{g}_{a}^{\alpha} & =-\frac{g^{\alpha \beta}}{\eta_{1}}(\kappa \lambda)_{\beta a} .
\end{aligned}
$$

A straightforward calculation gives the following expressions for the inverse metric $\left(\bar{g}^{-1}\right)^{\Lambda \bar{\Sigma}}$

$$
\begin{aligned}
\left(\bar{g}^{-1}\right)_{\alpha \beta} & =\left(g^{-1}\right)_{\alpha \beta}+\frac{1}{4 \eta_{1}^{2}}(\kappa \lambda \lambda)_{\alpha}(\kappa \lambda \lambda)_{\beta}+\frac{\left(G^{-1}\right)^{a b}}{\eta_{1}}(\kappa \lambda)_{\alpha a}(\kappa \lambda)_{\beta b}, \\
\left(\bar{g}^{-1}\right)^{00} & =\eta_{1}^{2} \\
\left(\bar{g}^{-1}\right)^{a b} & =\lambda^{a} \lambda^{b}+\eta_{1}\left(G^{-1}\right)^{a b}, \\
\left(\bar{g}^{-1}\right)^{a 0} & =\eta_{1} \lambda^{a} \\
\left(\bar{g}^{-1}\right)^{0}{ }_{\alpha} & =\frac{1}{2}(\kappa \lambda \lambda)_{\alpha}, \\
\left(\bar{g}^{-1}\right)_{\alpha}^{a} & =\frac{\lambda^{a}}{2 \eta_{1}}(\kappa \lambda \lambda)_{\alpha}+\left(G^{-1}\right)^{a c}(\kappa \lambda)_{\alpha c .} .
\end{aligned}
$$

We then find

$$
\frac{\partial K}{\partial \Re \eta^{\Lambda}}\left(\bar{g}^{-1}\right)^{\Lambda \Sigma} \frac{\partial K}{\partial \Re \eta^{\Sigma}}=4
$$

From this we see that the Kähler potentials obey a no scale type condition and thus give rise to positive semi-definite potentials * Later on, we will show that some of the Kähler

${ }^{*}$ The results in this section were also found in 1, 2], using a different coordinate system. 
spaces described in this section do factorize, namely when one performs the truncation on the homogeneous very special quaternionic spaces. In this case, a $S U(1,1) / U(1)$ part splits off and the remaining part gives rise to flat potentials.

\section{Analysis of the isometries}

We analyze which isometries of the special quaternionic manifold survive the truncation. As is discussed extensively in [7, the isometries of a special quaternionic manifold consist of the duality transformations inherited from the special Kähler space under the c-map, which are characterized by parameters $\omega^{i}$, and in addition, $2 n+4$ extra isometries characterized by $\epsilon^{0}, \epsilon^{+}, \alpha^{I}, \beta_{I}$. There are also hidden symmetries, which can exist when certain conditions are satisfied by the quaternionic manifold, characterized by $\hat{\alpha}^{I}, \hat{\beta}_{I}, \epsilon^{-}$. It was proved in [7] that all hidden symmetries are realized if and only if the quaternionic manifold is symmetric. For convenience, we repeat here the results of [7] concerning the infinitesimal transformations (note that we have redefined $D=\frac{1}{2} \log \phi$ )

$$
\begin{aligned}
\delta \phi= & \phi\left(-\epsilon^{0}+2 \sigma \epsilon^{-}+\hat{\alpha}^{I} B_{I}-\hat{\beta}_{I} A^{I}\right) \\
\delta \sigma= & \epsilon^{+}+\frac{1}{2}\left(\alpha^{I} B_{I}-\beta_{I} A^{I}\right)+\left(\sigma^{2}-\phi^{2}\right) \epsilon^{-}+\sigma\left(\frac{1}{2} \hat{\alpha}^{I} B_{I}-\frac{1}{2} \hat{\beta}_{I} A^{I}-\epsilon^{0}\right)+\mathcal{D} h \\
\delta A^{I}= & \alpha^{I}+\sigma \hat{\alpha}^{I}+B^{I}{ }_{J}(\omega) A^{J}-D^{I J}(\omega) B_{J} \\
& +\left(\epsilon^{-} \sigma+\frac{1}{2} \hat{\alpha}^{J} B_{J}-\frac{1}{2} \hat{\beta}_{J} A^{J}-\frac{1}{2} \epsilon^{0}\right) A^{I}-\partial^{I} \mathcal{D}\left(h+\frac{1}{2} \phi z_{2}\right) \\
\delta B_{I}= & \beta_{I}+\sigma \hat{\beta}_{I}+C_{I J}(\omega) A^{J}-B_{I}^{J}(\omega) B_{J} \\
& +\left(\epsilon^{-} \sigma+\frac{1}{2} \hat{\alpha}^{J} B_{J}-\frac{1}{2} \hat{\beta}_{J} A^{J}-\frac{1}{2} \epsilon^{0}\right) B_{I}+\partial_{I} \mathcal{D}\left(h+\frac{1}{2} \phi z_{2}\right) \\
\delta X^{I}= & B_{J}^{I}(\omega) X^{J}+\frac{1}{2} i D^{I J}(\omega) F_{J} \\
& +\mathcal{D}\left(-\frac{1}{2} i \overline{\mathcal{B}}^{I}\left(X^{J} \mathcal{B}_{J}\right)+\frac{1}{16} i\left(N^{-1}\right)^{I J} \mathcal{B}^{K} \bar{F}_{J K L} \mathcal{B}^{L}(X N \bar{X})\right)
\end{aligned}
$$

where

$$
\mathcal{D} h=\left(\epsilon^{-}+\hat{\alpha}^{I} \frac{\partial}{\partial A^{I}}+\hat{\beta}_{I} \frac{\partial}{\partial B_{I}}\right) h,
$$

the function $z$ is given by the expression:

$$
z_{2} \equiv \mathcal{B}_{I} \overline{\mathcal{B}}^{I}-2 \frac{\left(X^{I} \mathcal{B}_{I}\right)\left(\bar{X}^{J} \overline{\mathcal{B}}_{J}\right)}{X N \bar{X}},
$$

and the function $h$ is the real function defined by

$$
\begin{aligned}
h(X, \bar{X}, A, B)=- & \frac{1}{16}\left\{\left(\mathcal{B}_{I} \overline{\mathcal{B}}^{I}\right)^{2}-\frac{1}{6}\left[\left(F_{I J K} \overline{\mathcal{B}}^{I} \overline{\mathcal{B}}^{J} \overline{\mathcal{B}}^{K}\right)\left(X^{L} \mathcal{B}_{L}\right)+\text { h.c. }\right]\right. \\
& \left.+\frac{1}{16}(X N \bar{X}) \overline{\mathcal{B}}^{I} \overline{\mathcal{B}}^{J} F_{I J K} N^{-1}{ }^{K L} \bar{F}_{L M N} \mathcal{B}^{M} \mathcal{B}^{N}\right\}
\end{aligned}
$$


with

$$
\begin{aligned}
\mathcal{B}_{I} & =B_{I}+\frac{1}{2} i F_{I J} A^{J} \equiv N_{I J} \mathcal{B}^{J} \\
N_{I J} & =\frac{1}{4}\left(F_{I J}+\bar{F}_{I J}\right)
\end{aligned}
$$

and $F(X)$ a holomorphic function which is homogeneous of second degree in the variables $X^{I}$. The subscripts $I, J, \ldots$ on $F$ denote differentiation with respect to $X^{I}, X^{J}$, etc. In these expressions, the fields $X^{I}$ are not independent complex fields. The independent fields are introduced through the ratios $z^{A}=X^{A} / X^{0}=\frac{1}{2}\left(x^{A}+i y^{A}\right)$ (with $z^{0}:=1$ ).

In the 'very special' case, $F(X)=i \frac{d_{A B C} X^{A} X^{B} X^{C}}{X^{0}}$, and the matrix elements $N_{I J}$ are given by

$$
N_{00}=\frac{1}{2} i(\kappa z z z)+h . c ., \quad N_{0 A}=-\frac{3}{4} i(\kappa z z)_{A}+h . c ., \quad N_{A B}=\frac{3}{2}(d y)_{A B} .
$$

For later use, we give also the truncated elements:

$$
\begin{aligned}
N_{\alpha \beta} & =-\frac{3}{2}(\kappa y)_{\alpha \beta}, \quad N_{a b}=-\frac{3}{2}(\kappa y)_{a b}, \\
N_{a \alpha} & =N_{\alpha 0}=0, \\
N_{a 0} & =\frac{3}{4}(\kappa x y)_{a}, \\
N_{00} & =\frac{1}{8}(\kappa y y y)-\frac{3}{8}(\kappa x x y) .
\end{aligned}
$$

The matrices $B, C$ and $D$ characterize the duality transformations, and in the 'very special' case, the matrix-elements are given explicitly by

$$
B_{J}^{I}=\left(\begin{array}{cc}
\beta & a_{B} \\
b^{A} & \tilde{B}_{B}^{A}+\frac{1}{3} \beta \delta_{B}^{A}
\end{array}\right), \quad C_{I J}=\left(\begin{array}{cc}
0 & 0 \\
0 & 3 d_{A B C} b^{C}
\end{array}\right), \quad D^{I J}=\left(\begin{array}{cc}
0 & 0 \\
0 & -\frac{4}{9} C^{A B C} a_{C}
\end{array}\right) .
$$

The parameters $b^{A}$ and $\beta$ correspond to isometries inherited from the special Kähler manifold under the c-map and are always present, while the parameters $a_{B}$ correspond to hidden symmetries. The elements $\tilde{B}_{B}^{A}$ correspond to the symmetries of $d_{A B C}$ (see [7] for details).

We also list the non-zero commutators between the generators of the isometries. First there are those not involving the duality transformations:

$$
\begin{array}{lll}
{\left[\underline{\epsilon}_{0}, \underline{\epsilon}_{ \pm}\right]= \pm \underline{\epsilon}_{ \pm},} & & {\left[\underline{\epsilon}_{-}, \underline{\epsilon}_{+}\right]=2 \underline{\epsilon}_{0},} \\
{\left[\underline{\epsilon}_{0}, \underline{\alpha}_{I}\right]=\frac{1}{2} \underline{\alpha}_{I},} & & {\left[\underline{\epsilon}_{0}, \underline{\hat{\alpha}}_{I}\right]=-\frac{1}{2} \underline{\hat{\alpha}}_{I},} \\
{\left[\underline{\epsilon}_{0}, \underline{\beta}^{I}\right]=\frac{1}{2} \underline{\beta}^{I},} & & {\left[\underline{\epsilon}_{0}, \underline{\hat{\beta}}^{I}\right]=-\frac{1}{2} \underline{\hat{\beta}}^{I},} \\
{\left[\underline{\epsilon}_{-}, \underline{\alpha}_{I}\right]=-\underline{\hat{\alpha}}_{I},} & & {\left[\underline{\epsilon}_{+}, \underline{\hat{\alpha}}_{I}\right]=\underline{\alpha}_{I},} \\
{\left[\underline{\epsilon}_{-}, \underline{\beta}^{I}\right]=-\underline{\hat{\beta}}^{I},} & & {\left[\underline{\epsilon}_{+}, \underline{\hat{\beta}}^{I}\right]=\underline{\beta}^{I},} \\
{\left[\underline{\alpha}_{I}, \underline{\beta}^{J}\right]=-\delta_{I}^{J} \underline{\epsilon}_{+},} & & {\left[\underline{\hat{\alpha}}_{I}, \underline{\hat{\beta}}^{J}\right]=-\delta_{I}^{J} \underline{\epsilon}_{-} .}
\end{array}
$$


The commutators involving the duality transformations are:

$$
\begin{aligned}
& {\left[\alpha^{I} \underline{\alpha}_{I}+\beta_{I} \underline{\beta}^{I}, \omega^{i} \underline{\omega}_{i}\right]=\alpha^{\prime I} \underline{\alpha}_{I}+\beta_{I}^{\prime}, \underline{\beta}^{I},} \\
& {\left[\hat{\alpha}^{I} \underline{\hat{\alpha}}_{I}+\hat{\beta}_{I} \underline{\hat{\beta}}^{I}, \omega^{i} \underline{\omega}_{i}\right]=\hat{\alpha}^{\prime} \underline{\hat{\alpha}}_{I}+\hat{\beta}^{\prime}{ }_{I} \underline{\hat{\beta}}^{I},}
\end{aligned}
$$

where

$$
\left(\begin{array}{c}
\alpha^{\prime I} \\
\beta_{J}^{\prime}
\end{array}\right)=\left(\begin{array}{cc}
B^{I} K(\omega) & -D^{I L}(\omega) \\
C_{J K}(\omega) & -B^{L}{ }_{J}(\omega)
\end{array}\right)\left(\begin{array}{c}
\alpha^{K} \\
\beta_{L}
\end{array}\right)
$$

and likewise for $\hat{\alpha}^{\prime}$ and $\hat{\beta}^{\prime}$. Finally

$$
\left[\alpha^{I} \underline{\alpha}_{I}+\beta_{I} \underline{\beta}^{I}, \hat{\alpha}^{I} \underline{\hat{\alpha}}_{I}+\hat{\beta}_{I} \underline{\hat{\beta}}^{I}\right]=\left(\alpha^{I} \hat{\beta}_{I}-\hat{\alpha}^{I} \beta_{I}\right) \underline{\epsilon}_{0}+\omega^{i}(\alpha, \beta, \hat{\alpha}, \hat{\beta}) \underline{\omega}_{i} .
$$

It can be shown that duality transformations corresponding to the parameters $\omega(\alpha, \beta, \hat{\alpha}, \hat{\beta})$ correspond to the matrices [7]

$$
\begin{aligned}
B_{J}^{I}(\alpha, \beta, \hat{\alpha}, \hat{\beta}) & =-\frac{1}{2}\left(\hat{\alpha}^{I} \beta_{J}+\alpha^{I} \hat{\beta}_{J}\right)-\partial^{I} \partial_{J} h^{\prime \prime}(\alpha, \beta, \hat{\alpha}, \hat{\beta}), \\
C_{I J}(\alpha, \beta, \hat{\alpha}, \hat{\beta}) & =-\hat{\beta}_{(I} \beta_{J}+\partial_{I} \partial_{J} h^{\prime \prime}(\alpha, \beta, \hat{\alpha}, \hat{\beta}), \\
D^{I J}(\alpha, \beta, \hat{\alpha}, \hat{\beta}) & =-\hat{\alpha}^{(I} \alpha^{J)}+\partial^{I} \partial^{J} h^{\prime \prime}(\alpha, \beta, \hat{\alpha}, \hat{\beta}),
\end{aligned}
$$

where

$$
\partial^{I}:=\frac{\partial}{\partial B_{I}}, \partial_{I}:=\frac{\partial}{\partial A^{I}}
$$

and

$$
h^{\prime \prime}(\alpha, \beta, \hat{\alpha}, \hat{\beta}) \equiv(\alpha \cdot \partial+\beta \cdot \partial)(\hat{\alpha} \cdot \partial+\hat{\beta} \cdot \partial) h(X, \bar{X}, A, B) .
$$

From analyzing the infinitesimal transformations, we can now find the remaining isometries after the truncation (2.3). We demand that fields that were put to zero remain zero after performing an infinitesimal transformation. One easily finds that the isometries corresponding to $\epsilon^{0}, \alpha^{a}$ and $\beta_{\alpha}$ survive the truncation. The truncation also restricts the possible duality transformations. For our convenience, we start by rewriting the variation of the fields $X^{A}$ as function of the $x^{A}$ and $y^{A}$. For the 'very special' case we obtain

$$
\begin{aligned}
& \frac{1}{2} \delta x^{A}=B_{0}^{A}+\frac{1}{2}\left(B^{A}{ }_{B}-B^{0}{ }_{0} \delta^{A}{ }_{B}\right) x^{B}-\frac{1}{4}\left(\frac{3}{2} D^{A B} d_{B C D}+B^{0}{ }_{C} \delta^{A}{ }_{D}\left(x^{C} x^{D}-y^{C} y^{D}\right)\right), \\
& \frac{1}{2} \delta y^{A}=\frac{1}{2}\left(B_{B}^{A}-B^{0}{ }_{0} \delta^{A}{ }_{B}\right) y^{B}-\frac{1}{4}\left(\frac{3}{2} D^{A B} d_{B C D}+B^{0}{ }_{C} \delta^{A}{ }_{D}\left(x^{C} y^{D}+y^{C} x^{D}\right)\right),
\end{aligned}
$$

disregarding for now the possible existence of any hidden symmetries. From this, and the variations of $A^{I}$ and $B_{I}$ we infer the following conditions on the $S p(2 n+2, \mathbb{R})$ matrix of the duality transformations:

$$
\begin{aligned}
& B^{\alpha}{ }_{0}=b^{\alpha}=0, \\
& B^{\alpha}{ }_{a}=B^{a}{ }_{\alpha}=0, \\
& D^{\alpha \beta}=D^{a b}=0 .
\end{aligned}
$$


From the last equations, using (2.4), (2.11), and (4.8) we can further deduce that

$$
\begin{aligned}
a_{\alpha} & =0, \\
C_{\alpha \beta} & =C_{a b}=0 .
\end{aligned}
$$

Finally, there are the possible hidden symmetries $\left\{\hat{\alpha}, \hat{\beta}, \epsilon_{-}\right\}$. For these, the analysis is more complicated. We start by demanding that $\mathcal{D} h=0$ after truncation, a condition that is necessary for the variation $\delta \sigma$ to vanish. From this condition, a short calculation based on results in [7] for the expression $\mathcal{D} h$ reveals that the hidden isometries $\hat{\alpha}^{a}, \hat{\beta}^{\alpha}$ and $\epsilon^{-}$are certainly broken. For the remaining isometries $\hat{\alpha}^{\beta}, \hat{\beta}^{a}$, we must check that they satisfy the following conditions (after truncation)

$$
\begin{aligned}
\partial^{\alpha} \mathcal{D} h & =\partial_{a} \mathcal{D} h=0, \\
\partial^{\alpha} z_{2} & =\partial_{a} z_{2}=0 .
\end{aligned}
$$

After some calculation, one finds that this is indeed the case. Finally, one can check that also the last term in the variation of $X^{I}$ vanishes when calculating the variations $\delta x^{\alpha}$ and $\delta y^{a}$ for the remaining isometries. Crucial here is the fact that the matrix elements $N_{\alpha a}$ and $N_{\alpha 0}$ vanish identically upon truncation (see (4.7)). We conclude that the remaining isometries are characterized by parameters $\left\{\alpha^{0}, \alpha^{a}, \hat{\alpha}^{\beta}, \beta_{\alpha}, \hat{\beta}_{0}, \hat{\beta}_{a}, \epsilon_{0}\right\}$ and by duality transformations satisfying (4.16) and (4.17). As an extra check one can verify, using (4.13), that the the duality transformations corresponding to the hidden symmetries also satisfy (4.16) and (4.17). Note that of course only hidden symmetries which were there in the first place, will remain after the truncation.

The same analysis can be done for the minimal coupling case, $F(X)=X^{I} X^{J} \eta_{I J}$, which gives rise to a class of symmetric special quaternionic spaces ${ }^{*}$. In this case, the duality transformations are not of the form shown in equation (4.8), but obey the following equations: $B_{I J}=-B_{J I}$, where $B_{I J} \equiv \eta_{I K} B_{J}^{K}$, and $C_{I J}=\frac{1}{4} \eta_{I K} D^{K L} \eta_{L J}$. This means the group of duality transformations is $U(n, 1)$, with the generators $B_{J}^{I}$ forming a $S O(n, 1)$ subgroup. Using the transformations (4.1), which are valid for all special quaternionic spaces, we find the following result after the truncation:

- broken isometries: $\left\{B_{0}^{\alpha}, B_{\alpha}^{0}, B_{\alpha}^{a}, B_{a}^{\alpha}, D^{\alpha \beta}, D^{a b}, C_{\alpha \beta}, C_{a b}, \epsilon_{-}, \epsilon_{+}, \hat{\alpha}^{0}, \hat{\alpha}^{a}, \alpha^{\beta}, \hat{\beta}_{\alpha}, \beta_{0}, \beta_{a}\right\}$

- remaining isometries: $\left\{\alpha^{0}, \alpha^{a}, \hat{\alpha}^{\beta}, \beta_{\alpha}, \hat{\beta}_{0}, \hat{\beta}_{a} \epsilon_{0}, B_{\beta}^{\alpha}, B_{b}^{a}, D^{\alpha a}, D^{a \alpha}, D^{\alpha 0}, D^{0 \alpha}, C_{\alpha a}, C_{a \alpha}, C_{\alpha 0}, C_{0 \alpha}\right\}$.

In the next section, we will take a closer look at the isometry algebra that arises when truncating homogeneous special quaternionic manifolds. For these manifolds, it is known which hidden symmetries are realized.

${ }^{*} \eta_{I J}$ is a constant diagonal matrix with eigenvalues $(1,-1,-1, \ldots,-1)$. 


\section{Truncation of homogeneous very special quaternionic-Kähler manifolds}

The complete classification of homogeneous very special quaternionic-Kähler manifolds (HVSQ Manifolds from now on) was performed by de Wit and Van Proeyen in [16], and the symmetry structure of the isometries was further studied in [7]. The solutions are denoted by $L(q, P)$, where $q$ and $P$ are integers with $q \geq-1$ and $P \geq 0$. For $q$ equal to a multiple of 4 , there exist additional solutions denoted by $L(4 m, P, \dot{P})$, with $m \geq 0$ and $P, \dot{P} \geq 1$. Here $L(4 m, P, \dot{P})=L(4 m, \dot{P}, P)$. The different solutions are listed in Table 1, where the new spaces discovered in [16] are indicated by a $\star$.

\begin{tabular}{|l|ccc|}
\hline & $\begin{array}{c}\text { Real } \\
\left(\operatorname{dim}_{\mathbb{R}}=n-1\right)\end{array}$ & $\begin{array}{c}\text { Kähler } \\
\left(\operatorname{dim}_{\mathbb{R}}=2 n\right)\end{array}$ & $\begin{array}{c}\text { Quaternionic } \\
\left(\operatorname{dim}_{\mathbb{R}}=4 n+4\right)\end{array}$ \\
\hline$L(-1,0)$ & $S O(1,1)$ & {$\left[\frac{S U(1,1)}{U(1)}\right]^{2}$} & $\frac{S O(3,4)}{(S U(2))^{3}}$ \\
& $\star$ & $\star$ \\
\hline$L(-1, P)$ & $\frac{S O(P+1,1)}{S O(P+1)}$ & {$\left[\frac{S U(1,1)}{U(1)}\right]^{3}$} & $\frac{S O(4,4)}{S O(4) \otimes S O(4)}$ \\
$L(0,0)$ & {$[S O(1,1)]^{2}$} & $\frac{S U(1,1)}{U(1)} \otimes \frac{S O(P+2,2)}{S O(P+2) \otimes S O(2)}$ & $\frac{S O(P+4,4)}{S O(P+4) \otimes S O(4)}$ \\
$L(0, P, \dot{P})$ & $\frac{S O(P+1,1)}{S O(P+1)} \otimes S O(1,1)$ & $W(P, \dot{P})$ \\
$L(q, P)$ & $Y(P, \dot{P})$ & $K(P, \dot{P})$ & $V(P, q)$ \\
$L(4 m, P, \dot{P})$ & $X(P, q)$ & $H(P, q)$ & $\star$ \\
$L(1,1)$ & $\star$ & $\star$ & $\frac{F_{4}}{U S p(6) \otimes S U(2)}$ \\
$L(2,1)$ & $\frac{S \ell(3, \mathbb{R})}{S O(3)}$ & $\frac{S p(6)}{U(3)}$ & $\frac{E_{6}}{S U(6) \otimes S U(2)}$ \\
$L(4,1)$ & $\frac{S \ell(3, \mathbb{C})}{S U(3)}$ & $\frac{S U(3,3)}{S U(3) \otimes S U(3) \otimes U(1)}$ & $\frac{E_{7}}{S O(12) \otimes S U(2)}$ \\
$L(8,1)$ & $\frac{S U^{*}(6)}{S p(3)}$ & $\frac{S O^{*}(12)}{S U(6) \otimes U(1)}$ & $\frac{E_{8}}{E_{7} \otimes S U(2)}$ \\
\hline
\end{tabular}

Table 1: Homogeneous special real spaces and their corresponding Kähler and quaternionic spaces. The integers $P, \dot{P}, q$ and $m$ can take all values $\geq 1$.

The symmetric spaces are the three varieties corresponding to $L(-1,0), L(0, P), L(1,1)$, $L(2,1), L(4,1)$, and $L(8,1)$, and the real spaces corresponding to $L(-1, P)$. For those cases the isometry group $G$ is given. For the non-symmetric spaces the isometry group $G$ is not semisimple, the isotropy group $H$ is always its maximal compact subgroup.

For HVSQ manifolds the components of $d_{A B C}$ can be brought into the canonical form 
with non-zero components

$$
d_{122}=1, \quad d_{1 \mu \nu}=-\delta_{\mu \nu}, \quad d_{2 i j}=-\delta_{i j}, \quad d_{\mu i j}=\gamma_{\mu i j}
$$

where the the coordinates $h^{A}$ are decomposed into $h^{1}, h^{2}, h^{\mu}$ and $h^{i}$, and the indices $\mu$ and $i$ run over $q+1$ and $r=(P+\dot{P}) \mathcal{D}_{q+1}$ values, respectively. Thus we have $n=3+q+r$. The coefficients $\gamma_{\mu i j}$ are the generators of a $(q+1)$-dimensional real Clifford algebra with positive signature, denoted by $\mathcal{C}(q+1,0)$,

$$
\gamma_{\mu i k} \gamma_{\nu k j}+\gamma_{\nu i k} \gamma_{\mu k j}=2 \delta_{i j} \delta_{\mu \nu}
$$

and $\mathcal{D}_{q+1}$ denotes the dimension of an irreducible representation of this Clifford algebra. In the case $q \neq 0 \bmod 4$, the irreducible representations for a given $q$ are unique and thus the gamma matrices are unique once we specify the number of irreducible representations, denoted by $P$. For $q=0 \bmod 4$ the representations $\gamma_{\mu}$ and $-\gamma_{\mu}$ are not equivalent, and a reducible representation is characterized by the multiplicity of each of these representations, $P$ and $\dot{P}$. Hence $r=(P+\dot{P}) \mathcal{D}_{q+1}$ in general. The symmetric spaces are characterized by $\Gamma_{i j k l}=0$, with $\Gamma_{i j k l}$ defined by

$$
\Gamma_{i j k l} \equiv \frac{3}{8}\left[\gamma_{\mu(i j} \gamma_{k l) \mu}-\delta_{(i j} \delta_{k l)}\right]
$$

For calculational purposes it's sometimes convenient to combine the indices 2 and $\mu$ in on index $M$. We can then rewrite the relations in section 5 . The non-zero components of the tensor $d_{A B C}$ are

$$
d_{1 M N}=-\eta_{M N}, \quad d_{M i j}=\gamma_{M i j}
$$

The $\gamma_{M i j}$ obey

$$
\gamma_{M i k} \gamma_{N}^{k j}+\gamma_{N i k} \gamma_{M}^{k j}=2 \delta_{i}^{j} \eta_{M N}
$$

with $\eta=(-1,1,1, \ldots, 1), \gamma_{\mu i j}=\gamma_{\mu}^{i j}$ and $\gamma_{2}^{i j}=-\gamma_{2 i j}=\delta_{i j}$. The symmetric spaces are then characterized by $\Gamma_{i j k l}=\frac{3}{8}\left[\gamma_{M(i j} \gamma_{k l) N} \eta^{M N}\right]=0$.

For the orientifold truncation, we take the index $\alpha$ of the previous sections to run over 1 and $M$ (this is necessary, considering the restriction (2.4) on the $d$-symbols), while the index $a$ is identified with the index $i^{*}$ (this can be generalized in some cases, as we will show in section 6.2). The symmetries of the $d$-symbols are encoded in the matrix $\tilde{B}^{A}{ }_{B}$, which appears in the duality transformations and has the following structure (see [7])

$$
\tilde{B}_{B}^{A}=\left(\begin{array}{ccc}
-2 \lambda & 0 & 2 \xi_{j} \\
0 & \lambda \delta_{N}^{M}+A^{M}{ }_{N} & -\zeta^{k} \gamma^{M}{ }_{k j} \\
-\zeta^{i} & \gamma_{N}{ }^{i k} \xi_{k} & -\frac{1}{2} \lambda \delta_{j}^{i}+\frac{1}{4} A^{P Q}\left(\gamma_{P Q}{ }^{i}{ }_{j}+S^{i}{ }_{j}\right.
\end{array}\right)
$$

The matrices $A^{M}{ }_{N}$ generate infinitesimal $S O(q+1,1)$-symmetries and $S^{i}{ }_{j}$ generate the group $\mathcal{S}_{q}(P, \dot{P})$ which is the centralizer of the Clifford algebra representation [7]. Now

\footnotetext{
${ }^{*}$ The case $(\alpha)=(1, M, i)$ was studied in $[8,9]$.
} 
using (4.16), we can see that all isometries will survive the orientifold truncation, except the ones with parameters $\xi_{j}$ and $\zeta^{j}$, which are broken. The full set of isometries of the HVSQ manifold consists of the above isometries coming from the symmetries of the $d$-symbols, plus the extra symmetries one gets from performing the r-map and $\mathbf{c}$ map. For non-symmetric HVSQ manifolds, the following hidden symmetries are realized: $\left\{\underline{a}^{M}, \underline{\hat{\alpha}}_{1}, \hat{\beta}^{M}, \hat{\beta}^{0}\right\}$. The analysis of the various commutation relations and the isometry algebras that arise, is performed in [7, resulting in the classification given in table [1. We will not repeat all the results here, and we refer to [7] for the details. Our aim is to study what happens to these manifolds upon performing the orientifold truncation. The important results are that the resulting Kähler manifolds are also homogenous manifolds that have one half of the dimension of the HVSQ manifold. Furthermore, they are always product manifolds of the form $\mathcal{M} \times S U(1,1) / U(1)$, with $\mathcal{M}$ having the same dimension as the original special Kähler space that is the target of the $\mathbf{c}$-map.

\subsection{Homogenity}

We have to show that the remaining isometries work transitively on the truncated manifold. It is obvious that this is so for the remaining shifts and scale transformation. To study the effect of the remaining duality transformations, we write down the truncated version of (4.15)

$$
\begin{aligned}
& \delta x^{a}=b^{a}+B^{a}{ }_{b} x^{b}-\beta x^{a}-\frac{3}{4} D^{a \alpha}\left(d_{\alpha c d} x^{c} x^{d}+d_{\alpha \beta \gamma} y^{\beta} y^{\gamma}\right)-\frac{1}{2} a_{c} x^{c} x^{a}, \\
& \delta y^{\alpha}=B^{\alpha}{ }_{\beta} y^{\beta}-\beta y^{\alpha}-\frac{3}{2} D^{\alpha b} d_{b c \gamma} x^{c} y^{\gamma}-\frac{1}{2} a_{c} x^{c} y^{\alpha} .
\end{aligned}
$$

Looking at the form of the matrix $\tilde{B}_{B}^{A}$ in (5.6), it is now clear that one can connect any two points in the truncated manifold by performing shifts $b^{a}$ (for the coördinates $x^{a}$ ), rotations $A^{M}{ }_{N}$ and scale transformations $\beta$ (for the coördinates $y^{\alpha}$ ). Thus, the truncated manifold is homogeneous. To find the elements of the isotropy group, we can choose a point with $A^{0}=A^{a}=B_{\alpha}=x^{a}=y^{\mu}=0$ (i.e. only $y^{1}, y^{2}$ different from 0 ). This point is left invariant by generators of $\mathcal{S}_{q}(P, \dot{P})$ and $S O(q+1)$, and by the generators of the hidden symmetries.

\subsection{Splitting of an $S U(1,1) / U(1)$-part}

We will show that there exists an $S U(1,1)$ - subalgebra of the isometry algebra, of which the three generators commute with all the other generators. First, we give a list of the isometries of the HVSQ spaces that survive the truncation:

$\left\{\underline{\lambda}, \underline{A}^{M}{ }_{N}, \underline{S}^{i}{ }_{j}, \underline{\beta}, \underline{\epsilon}_{0}, \underline{\beta}^{M}, \underline{\alpha}_{i}, \underline{b}_{i}, \underline{\hat{\alpha}}_{1}, \underline{\hat{\beta}}^{0}, \underline{\alpha}_{0}, \underline{\beta}^{1}\right\}$.

Next, we write down the commutators between the generators $\underline{\alpha}, \beta, \underline{a}$ and $\underline{b}$ which were not given explicitly in [7]. Using (4.12), one finds the following relations

$$
\begin{array}{ll}
{\left[\underline{\alpha}_{0}, \underline{\hat{\alpha}}_{\beta}\right]=0,} & {\left[\underline{\hat{\beta}}^{0}, \underline{\alpha}_{a}\right]=\underline{b}_{a},} \\
{\left[\underline{\alpha}_{0}, \underline{\hat{\beta}}^{b}\right]=-\underline{a}^{b},} & {\left[\underline{\hat{\beta}}^{0}, \underline{\beta}^{\alpha}\right]=0,}
\end{array}
$$




$$
\begin{aligned}
& {\left[\underline{\alpha}_{0}, \underline{\hat{\beta}}^{0}\right]=\underline{\epsilon}_{0}-\frac{3}{2} \underline{\beta}^{b},} \\
& {\left[\underline{\alpha}_{a}, \underline{\hat{\alpha}}_{\beta}\right]=3 d_{\beta a b} \underline{a}^{b},} \\
& {\left[\underline{\beta}^{\alpha}, \underline{\hat{\beta}}^{a}\right]=-\frac{4}{9} C^{\alpha a b} \underline{b}_{b},} \\
& {\left[\bar{\beta}^{1}, \hat{\hat{\alpha}}_{1}\right]=-\underline{\epsilon}_{0}+\frac{2}{3} \underline{\lambda}-\frac{1}{2} \underline{\beta},} \\
& {\left[\bar{\beta}^{2}, \hat{\hat{\alpha}}_{2}\right]=-\underline{\epsilon}_{0}-\frac{1}{3} \underline{\lambda}-\frac{1}{2} \underline{\beta},} \\
& {\left[\bar{\beta}^{\mu}, \underline{\hat{\alpha}}_{\nu}\right]=-\underline{A}_{\mu \nu}+\delta_{\mu \nu}\left(-\underline{\epsilon}_{0}-\frac{1}{3} \underline{\lambda}-\frac{1}{2} \underline{\beta}\right),} \\
& {\left[\underline{a}^{b}, \underline{\hat{\beta}}^{0}\right]=\hat{\beta}^{b}, \quad\left[\underline{b}_{a}, \underline{\alpha}_{0}\right]=-\underline{\alpha}_{a},} \\
& \left.\left[\underline{a}^{b}, \underline{\hat{\alpha}}_{\beta}\right]=0, \quad \underline{\hat{b}}_{a}, \underline{\alpha}_{b}\right]=-3 d_{\alpha a b} \underline{\beta}^{\alpha}, \\
& {\left[\underline{a}^{b}, \underline{\hat{\beta}}^{a}\right]=-\frac{4}{9} C^{\beta a b} \underline{\alpha}_{\beta}, \quad\left[\underline{b}_{a}, \underline{\beta}^{\alpha}\right]=0 .}
\end{aligned}
$$

When one now considers the explicit form of the $d$-symbols and the tensor $C^{A B C}$ ([7]), one finds that the set of generators $\left\{\underline{\hat{\alpha}}_{1}, \underline{\beta}^{1}, \underline{\epsilon} \equiv-\underline{\epsilon}_{0}+\frac{2}{3} \underline{\lambda}-\frac{1}{2} \underline{\beta}\right\}$ generates an $S U(1,1)$ subalgebra, and furthermore commutes with all other generators. The Kähler space resulting from the truncation will thus contain a $S U(1,1) / U(1)$-part which splits off from the manifold, with the $U(1)$ subgroup generated by the compact generator $\hat{\alpha}_{1}$. This result will be confirmed when we analyze the metric and curvature tensor later on.

\section{Truncation of symmetric special quaternionic- Kähler manifolds}

\subsection{SVSQ manifolds: the generic truncation}

In the previous section, we showed that a truncated HVSQ manifold takes the form $\mathcal{M} \times S U(1,1) / U(1)$. It is therefore interesting to treat the manifold $\mathcal{M}$ as a 'dual' Kähler manifold in the sense of [8], and to look closer at its properties. We shall start by looking at the subclass of symmetric very special quaternionic-Kähler manifolds (SVSQ manifolds). These manifolds take the form of coset spaces $G / H$, where the isometry group $G$ is a semisimple Lie-group, and the isotropy group $H$ is its maximal compact subgroup. The SVSQ manifolds are the ones denoted by $L(-1,0), L(0, P), L(1,1), L(2,1), L(4,1)$, and $L(8,1)$ in table (11). For these manifolds, all hidden symmetries are realized. The full set of isometries and the commutators are given in table 2, which we have taken from [7], completed with the additional hidden symmetries.

The isometry algebra can be decomposed as follows (see [7])

$$
\begin{aligned}
\mathcal{V} & =\mathcal{V}_{0}+\mathcal{V}_{1}+\mathcal{V}_{2}+\mathcal{V}_{-1}+\mathcal{V}_{-2}, \\
\mathcal{V}_{0} & =\underline{\epsilon}^{\prime} \oplus s o(q+3,3) \oplus \mathcal{S}_{q}(P, \dot{P}), \\
\mathcal{V}_{1} & =\left(\underline{\xi}^{i}, \underline{b}_{i}\right) \oplus\left(\underline{\alpha}_{i}, \underline{\beta}^{i}\right)=(1, s, v), \\
\mathcal{V}_{2} & =\underline{\epsilon}_{+} \oplus\left(\underline{\alpha}_{1}, \underline{\beta}^{M}, \underline{\beta}^{0}\right) \oplus \underline{b}_{1}=(2, v, 0), \\
\mathcal{V}_{-1} & =\left(\underline{a}^{i}, \underline{\zeta}_{i}\right) \oplus\left(\underline{\hat{\alpha}}_{i}, \underline{\hat{\beta}}^{i}\right)=(-1, s, v), \\
\mathcal{V}_{-2} & =\underline{\epsilon}_{-} \oplus\left(\underline{\hat{\alpha}}_{0}, \underline{\hat{\alpha}}_{M}, \underline{\hat{\beta}}^{1}\right) \oplus \underline{a}^{1}=(-2, v, 0),
\end{aligned}
$$


Table 2: Roots of the isometries of the symmetric very special quaternionic spaces: so $(q+2,2)$ is generated by $\left\{\underline{A}^{M}{ }_{N}, \underline{a}^{M}, \underline{b_{M}}, \underline{\beta}-\frac{1}{3} \underline{\lambda}\right\}$ and $\lambda^{\prime} \equiv \frac{2}{3} \lambda+\beta$

\begin{tabular}{||l|ccrr|rr||}
\hline generator & $\mathcal{S}_{q}$ & $s o(q+2,2)$ & $\underline{\lambda}^{\prime}$ & $\underline{\epsilon}_{0}$ & $\underline{\lambda}^{\prime}-2 \underline{\epsilon}_{0}$ & $\underline{\lambda}^{\prime}+2 \underline{\epsilon}_{0}$ \\
\hline$\underline{\epsilon}_{+}$ & 0 & 0 & 0 & 1 & -2 & 2 \\
$\left(\underline{\alpha}_{1}, \underline{\beta}^{M}, \underline{\beta}^{0}\right)$ & 0 & $v$ & 1 & $\frac{1}{2}$ & 0 & 2 \\
$\underline{b}_{1}$ & 0 & 0 & 2 & 0 & 2 & 2 \\
$\left(\underline{\alpha}_{i}, \underline{\beta}^{i}\right)$ & $v$ & $s$ & 0 & $\frac{1}{2}$ & -1 & 1 \\
$\left(\underline{\xi}^{i}, \underline{b}_{i}\right)$ & $v$ & $\bar{s}$ & 1 & 0 & 1 & 1 \\
$\left(\underline{\hat{\alpha}}_{1}, \underline{\hat{\beta}}^{M}, \underline{\hat{\beta}}^{0}\right)$ & 0 & $v$ & 1 & $-\frac{1}{2}$ & 2 & 0 \\
$\left(\underline{\alpha}_{0}, \underline{\alpha}_{M}, \underline{\beta}^{1}\right)$ & 0 & $v$ & -1 & $\frac{1}{2}$ & -2 & 0 \\
\hline$\underline{\epsilon}_{-}$ & 0 & 0 & 0 & -1 & 2 & -2 \\
$\left(\underline{\hat{\alpha}}_{0}, \underline{\hat{\alpha}}_{M}, \underline{\hat{\beta}}^{1}\right)$ & 0 & $v$ & -1 & $-\frac{1}{2}$ & 0 & -2 \\
$\underline{a}^{1}$ & 0 & 0 & -2 & 0 & -2 & -2 \\
$\left(\underline{\hat{\alpha}}_{i}, \underline{\hat{\beta}}^{i}\right)$ & $v$ & $\bar{s}$ & 0 & $-\frac{1}{2}$ & 1 & -1 \\
$\left(\underline{\zeta}_{i}, \underline{a}^{i}\right)$ & $v$ & $s$ & -1 & 0 & -1 & -1 \\
\hline
\end{tabular}

where the representations of $\mathcal{V}_{1}, \mathcal{V}_{2}, \mathcal{V}_{-1}$ and $\mathcal{V}_{-2}$ are indicated according to the three subalgebras of $\mathcal{V}_{0}$ and $\underline{\epsilon}^{\prime} \equiv 2 \underline{\epsilon}_{0}+\underline{\beta}+\frac{2}{3} \underline{\lambda}$.

After the orientifold projection, the following set of isometries will survive:

$\left\{\underline{\lambda}, \underline{A}^{M}{ }_{N}, \underline{S}^{i}{ }_{j}, \underline{\beta}, \underline{\epsilon}_{0}, \underline{\beta}^{M}, \underline{\alpha}_{i}, \underline{b}_{i}, \underline{\hat{\alpha}}_{1}, \underline{\hat{\beta}}^{0}, \underline{\alpha}_{0}, \underline{\beta}^{1}, \underline{a}^{i}, \underline{\hat{\beta}}^{i}, \underline{\hat{\alpha}}_{M}\right\}$.

Counting the number of compact and non-compact generators gives us:

- $\operatorname{dim} G=2 n+6+\frac{1}{2} q(q+3)+2 r+\operatorname{dim} \mathcal{S}_{q}$ remaining isometries ,

- $\operatorname{dim} H=n+1+\frac{1}{2} q(q+1)+r+\operatorname{dim} \mathcal{S}_{q}$ remaining compact isometries ,

so that the dimension of the truncated manifolds is given by

$$
\operatorname{dim} \mathcal{M}_{\text {trunc }}=\operatorname{dim} G-\operatorname{dim} H=n+r+q+5=2 n+2 .
$$

This is consistent with the result $\mathcal{M}_{\text {trunc }}=\mathcal{K}_{\text {dual }} \times S U(1,1) / U(1)$. Also, looking at the root diagrams presented in [7] and [17] reveals the following fact: if a root corresponds to an isometry that is projected out, the opposite root also belongs to a broken isometry. In other words, opposing roots are always projected out together.

After performing the truncation on the isometry algebra (6.1) and throwing away the 
$S U(1,1) / U(1)$ part that splits off, we find the following structure for the isometry algebra of $\mathcal{K}_{\text {dual }}$

$$
\begin{aligned}
& \mathcal{W}=\mathcal{W}_{0}+\mathcal{W}_{1}+\mathcal{W}_{2}+\mathcal{W}_{-1}+\mathcal{W}_{-2}, \\
& \mathcal{W}_{0}=\underline{\epsilon}^{\prime} \oplus\left(\underline{\epsilon}_{0}-\frac{3}{2} \underline{\beta}, \underline{\alpha}_{0}, \underline{\beta}_{0}\right) \oplus s o(q+1,1) \oplus \mathcal{S}_{q}(P, \dot{P}), \\
& \mathcal{W}_{1}=\underline{b}^{i} \oplus \underline{\alpha}_{i}=(1, s, s, v) \\
& \mathcal{W}_{2}=\underline{\beta}^{M}=(2,0, v, 0) \\
& \mathcal{W}_{-1}=\underline{a}_{i} \oplus \underline{\hat{\beta}}^{i}=(-1, s, s, v), \\
& \mathcal{W}_{-2}=\underline{\hat{\alpha}}_{M}=(-2,0, v, 0) .
\end{aligned}
$$

From this, we see that the generators $\underline{\epsilon}^{\prime}, \underline{\beta}^{M}, \underline{\hat{\alpha}}_{M}$, together with the generators $\underline{A}_{M N}$ of $s o(q+1,1)$ form the algebra $s o(q+2,2)$. We can then decompose the isometry algebra with respect to the grading under $\underline{\epsilon}_{0}-\frac{3}{2} \underline{\beta}$

$$
\begin{aligned}
& \mathcal{W}^{\prime}=\mathcal{W}_{0}^{\prime}+\mathcal{W}_{1}^{\prime}+\mathcal{W}_{2}^{\prime}+\mathcal{W}_{-1}^{\prime}+\mathcal{W}_{-2}^{\prime}, \\
& \mathcal{W}_{0}^{\prime}=\underline{\epsilon}_{0}-\frac{3}{2} \underline{\beta} \oplus s o(q+2,2) \oplus \mathcal{S}_{q}(P, \dot{P}), \\
& \mathcal{W}_{1}^{\prime}=\underline{a}^{i} \oplus \underline{\alpha}_{i}=(1, s, v) \\
& \mathcal{W}_{2}^{\prime}=\underline{\alpha}_{0}=(2,0,0), \\
& \mathcal{W}_{-1}^{\prime}=\underline{b}_{i} \oplus \underline{\hat{\beta}}^{i}=(-1, s, v), \\
& \mathcal{W}_{-2}^{\prime}=\underline{\hat{\beta}}_{0}=(-2,0,0) .
\end{aligned}
$$

This has exactly the same structure as the isometry algebra of the symmetric very special Kähler spaces (see [7]). The isotropy group is again the maximal compact subgroup of the isometry group. Another way to get this result, is by looking at all possible semi-simple subgroups of the isometry groups of the symmetric very special quaternionic manifolds and imposing the constraints that these subgroups have the right dimension and the right number of non-compact generators. One then always finds a unique possibility.

From these results, we can conclude that the dual Kähler spaces are the same cosets as the original special Kähler spaces. Note that this result does not necessarily imply that the dual space has the same curvature. We will investigate this in the next section.

\subsection{A generalisation for the case $L(0, P)$}

When $P>1$, we can split the index $i$ in two parts, $(i)=\left(i_{+}, i_{-}\right)$where $i_{+}$runs over $P_{+} \mathcal{D}_{q+1}$ values, $i_{-}$runs over $P_{-} \mathcal{D}_{q+1}$ values, $P=P_{+}+P_{-}$, and in such a way that $\gamma_{\mu i_{+} i_{-}}=0$. We can take the $\alpha$-index of the previous sections to run over $1,2, \mu$ and $i_{+}$, and identify the $a$-index with $i_{-}$. Therefore, from now on, we will write the $i_{+}$-index as $\tilde{M}$ and the $i_{-}$-index as $a$. We can then start with the isometry algebra for the very special quaternionic spaces given in (6.1), specialised to the case $q=\dot{P}=0$, and with $(i)=(\tilde{M}, a)$. After the truncation, the $S U(1,1)$ generated by $\left\{\underline{\hat{\alpha}}_{1}, \underline{\beta}^{1}, \underline{\epsilon} \equiv-\underline{\epsilon}_{0}+\frac{2}{3} \underline{\lambda}-\frac{1}{2} \underline{\beta}\right\}$, 
does not split off anymore if $P_{+} \neq 0$. We find the following structure for the truncated isometry algebra

$$
\begin{aligned}
& \mathcal{W}=\mathcal{W}_{0}+\mathcal{W}_{+}+\mathcal{W}_{-}, \\
& \mathcal{W}_{0}=S O(2,2)_{+} \oplus S O\left(P_{+}\right) \oplus S O(2,2)_{-} \oplus S O\left(P_{-}\right), \\
& \mathcal{W}_{+}=\left(\underline{\xi}^{\tilde{M}}, \underline{\hat{\alpha}}_{\tilde{M}}, \underline{\beta}^{\tilde{M}}, \underline{\zeta}_{\tilde{M}}\right)=(v, v, 0,0), \\
& \mathcal{W}_{-}=\left(\underline{a}^{b}, \underline{\alpha}_{b}, \underline{b}_{a}, \underline{\hat{\beta}}^{a}\right)=(0,0, v, v),
\end{aligned}
$$

where

- $S O(2,2)_{+}$is generated by $\left\{\underline{\hat{\alpha}}_{1}, \underline{\beta}^{1}, \underline{\epsilon}, \underline{\beta}^{2}+\underline{\beta}^{3}, \underline{\alpha}^{2}+\underline{\alpha}^{3},-\underline{\epsilon}-2 \underline{A}_{3}^{2}\right\}$,

- $S O(2,2)_{-}$by $\left\{\underline{\hat{\beta}}^{0}, \underline{\alpha}_{0}, \underline{\epsilon}_{0}-\frac{3}{2} \underline{\beta}, \underline{\beta}^{2}-\underline{\beta}^{3}, \underline{\alpha}^{2}-\underline{\alpha}^{3},-\underline{\epsilon}+2 \underline{A}_{3}^{2}\right\}$,

- $S O\left(P_{+}\right)$by $S_{\tilde{N}}^{\tilde{M}}$ and $S O\left(P_{-}\right)$by $S_{b}^{a}$.

It is also easy to see that the generators in $W_{+}$commute with those in $W_{-}$, since their commutators will be proportional to either $\delta^{a \tilde{M}}, \gamma^{\mu a \tilde{M}}$ or $S^{a \tilde{M}}$ and those are all zero. Thus, we find that the isometry group surviving the truncation is $S O\left(P_{+}+2,2\right) \times S O\left(P_{-}+2,2\right)$. It is again straightforward to show that these isometries work transitively on the manifold, so that the resulting Kähler space is*

$$
\frac{S O\left(P_{+}+2,2\right)}{S O\left(P_{+}+2\right) \times S O(2)} \times \frac{S O\left(P_{-}+2,2\right)}{S O\left(P_{-}+2\right) \times S O(2)} .
$$

\subsection{The other SSQ manifolds}

Finally, we can try to extend our classification by analyzing the effect of the truncation on symmetric special quaternionic manifolds that are not very special. There are three cases to consider: (see [17] for an overview)

- The space $U(1,2) /(U(1) \times U(2))$, which one gets by acting with the c-map on the empty special Kähler space.

- The space $G_{2} /(S U(2) \times S U(2))$, resulting from the c-map on the special Kähler manifold $S U(1,1) / U(1)$ that is characterized by the prepotential $F=i\left(X^{1}\right)^{3} / X^{0}$.

- The spaces denoted by $L(-2, P)$ in [17, resulting from the c-map on $U(P+1,1) /(U(P+1) \times U(1))$ : Kähler spaces characterized by a quadratic prepotential $F(X)=X^{I} \eta_{I J} X^{J}$. Because of the quadratic prepotential, the truncation conditions from [12] are trivially satisfied, so that no extra conditions need to be imposed. This means that different truncations are possible, depending on how we split the index $I$ into indices $(0, a, \alpha)$.

${ }^{*}$ For $P_{+}=0$, which was discussed in section 6.1] or for $P_{-}=0$, which was discussed in [8, 9] this reduces to $[S U(1,1) / U(1)]^{2} \times[S O(P+2,2) /(S O(P+2) \times S O(2))]$. 
The analysis of the duality symmetries is performed in [18] and [19], to which we refer for details. These are the results for the truncated manifolds *

- In the first case, the coset $S U(1,1) / U(1)$, the set of three surviving isometries being $\left\{\underline{\alpha}_{0}, \underline{\hat{\beta}}^{0}, \underline{\epsilon}_{0}\right\}$.

- In the second case, the coset $(S U(1,1) / U(1))^{2}$, with the two commuting $S U(1,1)$ subalgebras generated by $\left\{\underline{\alpha}_{0}, \underline{\hat{\beta}}^{0}, \underline{\epsilon}_{0}-\frac{3}{2} \underline{\beta}\right\}$ and $\left\{\underline{\hat{\alpha}}_{1}, \underline{\beta}^{1},-\underline{\epsilon}_{0}-\frac{1}{2} \underline{\beta}\right\}$. (Although the space is usually not considered to be 'very special', the duality transformations are still of the form (4.8).)

- In the third case, the structure of the isometry algebra before the truncation is

$$
\begin{aligned}
\mathcal{V} & =\mathcal{V}_{0}+\mathcal{V}_{\frac{1}{2}}+\mathcal{V}_{1}+\mathcal{V}_{-\frac{1}{2}}+\mathcal{V}_{-1}, \\
\mathcal{V}_{0} & =\underline{\epsilon}_{0} \oplus U(P+1,1), \\
\mathcal{V}_{\frac{1}{2}} & =\left(\underline{\alpha}_{I}, \underline{\beta}^{I}\right)=\left(\frac{1}{2}, P+2\right) \oplus\left(\frac{1}{2}, \overline{P+2}\right), \\
\mathcal{V}_{1} & =\epsilon^{+}, \\
\mathcal{V}_{-\frac{1}{2}} & =\left(\underline{\hat{\alpha}}_{I}, \underline{\hat{\beta}}^{I}\right)=\left(-\frac{1}{2}, P+2\right) \oplus\left(-\frac{1}{2}, \overline{P+2}\right), \\
\mathcal{V}_{-1} & =\epsilon^{-},
\end{aligned}
$$

forming $S U(P+2,2)$. For all possible truncations, we find the same result: a total number of $\frac{1}{2}(P+4)(P+3)$ isometries is preserved, of which $2(P+2)$ are non-compact. The truncated isometry algebra has the following structure:

$$
\begin{aligned}
\mathcal{V} & =\mathcal{V}_{0}+\mathcal{V}_{\frac{1}{2}}+\mathcal{V}_{-\frac{1}{2}}, \\
\mathcal{V}_{0} & =\underline{\epsilon}_{0} \oplus S O(P+1,1), \\
\mathcal{V}_{\frac{1}{2}} & =\left(\underline{\alpha}_{0}, \underline{\alpha}_{a}, \underline{\beta}^{\alpha}\right)=\left(\frac{1}{2}, v\right), \\
\mathcal{V}_{-\frac{1}{2}} & =\left(\underline{\hat{\alpha}}_{0}, \underline{\hat{\alpha}}_{a}, \underline{\hat{\beta}}^{\alpha}\right)=\left(-\frac{1}{2}, v\right),
\end{aligned}
$$

forming $S O(P+2,2)$. From this we conclude that the truncated spaces consist of the class $S O(P+2,2) /(S O(P+2) \times S O(2))$. These are symmetric Kähler spaces that are not special (see [20] for the classification). Note that no $S U(1,1)$-part splits off.

The results for the truncation of all SSQ manifolds are summarized in table 3 .

* As before the remaining isometries work transitively, so that we again find homogeneous Kähler submanifolds of the corresponding quaternionic spaces. 
Table 3: Truncation of symmetric special quaternionic manifolds

\begin{tabular}{|l|c|c|}
\hline & $\mathcal{M}_{Q}\left(\operatorname{dim}_{\mathbb{R}}=4 n+4\right)$ & $\mathcal{M}_{K H}\left(\operatorname{dim}_{\mathbb{R}}=2 n+2\right)$ \\
\hline$S G_{4}$ & $\frac{U(2,1)}{U(2) \times U(1)}$ & $\frac{S U(1,1)}{U(1)}$ \\
$L(-2, P)$ & $\frac{S U(P+2,2)}{S U(P+2) \times S U(2) \times U(1)}$ & $\frac{S O(P+2,2)}{S O(P+2) \times S O(2)}$ \\
$L(-1,0)$ & $\left.\frac{S O(3,4)}{(S U(2)}\right)$ & {$\left[\frac{S U(1,1)}{U(1)}\right]^{3}$} \\
$L(0,0)$ & $\frac{S O(4,4)}{S O(4) \times S O(4)}$ & {$\left[\frac{S U(1,1)}{U(1)}\right]^{4}$} \\
$L(0, P)$ & $\frac{S O(P+4,4)}{S O(P+4) \times S O(4)}$ & $\frac{S O\left(P_{+}+2,2\right)}{S O\left(P_{+}+2\right) \times S O(2)} \times \frac{S O(P-+2,2)}{S O(P-+2) \times S O(2)}$ \\
$S G_{5}$ & $\frac{G_{2(2)}}{S O(4)}$ & {$\left[\frac{S U(1,1)}{U U(1)}\right]^{2}$} \\
$L(1,1)$ & $\frac{F_{4(4)}}{U S p(6) \times U S p(2)}$ & $\frac{S U(1,1)}{U(1)} \times \frac{S p(6)}{U(3)}$ \\
$L(2,1)$ & $\frac{E_{6(2)}}{S U(6) \times S U(2)}$ & $\frac{S U(1,1)}{U(1)} \times \frac{S U(3,3)}{S U(3) \times S U(3) \times U(1)}$ \\
$L(4,1)$ & $\frac{E_{7(-8)}}{S O(12) \times S U(2)}$ & $\frac{S U(1,1)}{U(1)} \times \frac{S O^{*}(12)}{U(6)}$ \\
$L(8,1)$ & $\frac{E_{8(-24)}}{E_{7} \times S U(2)}$ & $\frac{S U(1,1)}{U(1)} \times \frac{E_{7(-26)}}{E_{6} \times S O(2)}$ \\
\hline
\end{tabular}

\section{$7 \quad$ Dual Kähler spaces}

In the previous sections we studied the result of the orientifold truncation (2.3) on the symmetric special quaternionic spaces. It was noticed in [8] that if one starts with the very special quaternionic spaces, characterized by a $d$-symbol $d_{A B C}$, and takes the truncation $(A)=(\alpha)$, that is $n_{-}=0$, the resulting Kähler spaces are always of the form $\mathcal{M}_{\text {trunc }}=S U(1,1) / U(1) \times \mathcal{K}_{\text {dual }}$. When we take orientifold truncations with $n_{-} \neq 0$, no $S U(1,1) / U(1)$ part splits off in general. However, for specific $d$-symbols* ${ }^{*}$ e.g those characterizing the HVSQ manifolds, we found a truncation with $n_{-} \neq 0$ where a $S U(1,1) / U(1)$ part splits off (see section [5.2). We can then, as in [8, 9, view the remaining manifold as a dual Kähler manifold. In the previous section, we found that in the symmetric case, the dual Kähler manifolds are the same cosets as the original very special Kähler spaces. In the first subsection, we will verify that the dual Kähler spaces also have the same curvature tensor in the symmetric case. For the non-symmetric case, the curvature tensors are different in general, as we will show by computing the scalar curvature for a specific case. Another important property to check is whether these dual Kähler spaces give rise to flat potentials, like in [8. We will find in the last subsection that this is indeed the case.

*The fact that in this case again a $S U(1,1) / U(1)$ part splits off does not depend on the fact that $d_{\mu i j}$ are $\gamma$-matrices and so there are also many non-homogeneous VSQ manifolds for which one can find truncations with $n_{-} \neq 0$ and a $S U(1,1) / U(1)$ part splitting off. 


\subsection{The curvature tensor of the truncated VSQ manifolds}

We start by looking at the scalar curvature. For the readers convenience, we have written down the explicit expressions for the components of the Christoffel connection and curvature tensor in the appendix.

We restrict ourselves to the homogeneous very special quaternionic manifolds. These are characterized by the $d$-symbols (5.1) mentioned in section 5. Inserting these $d$-symbols in the expressions for the metric (3.12) *, it is easy to see that in this case, the Kähler

manifolds have an $\frac{S U(1,1)}{U(1)}$ part that splits off. Indeed, we find $\bar{g}^{1 M}=\bar{g}^{1}{ }_{a}=\bar{g}^{1}{ }_{0}=0$, and therefore also, since the metric is Kähler, that $\bar{g}^{11}$ only depends on $t_{1}$ and that all other components are independent of $t_{1}$. We can now calculate the scalar curvature of the 'dual' Kähler space. We have

$$
\bar{R}=\bar{R}_{M}^{M}{ }_{N}^{N}+\bar{R}_{a b}^{a b}+\bar{R}_{0}{ }^{0}{ }_{0}^{0}+2 \bar{R}_{M a}^{M}+2 \bar{R}_{M 0}^{M}+2 \bar{R}_{a}^{a}{ }^{a}{ }^{0},
$$

with (see appendix)

$$
\begin{aligned}
& \bar{R}^{M}{ }_{M}{ }^{N}{ }_{N}=-(q+2)^{2}, \quad \bar{R}_{0}^{0}{ }_{0}^{0}=-2, \quad \bar{R}_{a 0}^{a}{ }_{0}^{0}=-r, \\
& \bar{R}_{a b}^{a b}=-r^{2}+\frac{1}{2} \eta^{M N} \gamma_{M f a} \gamma_{N b e} \frac{\left(\gamma_{K}^{a e} \chi^{K}\right)\left(\gamma_{L}^{b f} \chi^{L}\right)}{\left(\chi^{P} \chi_{P}\right)}+\frac{1}{2} \eta^{M N} \gamma_{M f b} \gamma_{N a e} \frac{\left(\gamma_{K}^{a e} \chi^{K}\right)\left(\gamma_{L}^{b f} \chi^{L}\right)}{\left(\chi^{P} \chi_{P}\right)} \text {, } \\
& \bar{R}_{M a}^{M}{ }^{a}=-r(q+2)+r-\frac{1}{2} \eta^{M N} \gamma_{M f a} \gamma_{N b e} \frac{\left(\gamma_{K}^{a e} \chi^{K}\right)\left(\gamma_{L}^{b f} \chi^{L}\right)}{\left(\chi^{P} \chi_{P}\right)}, \quad \bar{R}^{M}{ }_{M 0}{ }^{0}=0
\end{aligned}
$$

where we used the following expressions ${ }^{\dagger}$

$$
\begin{aligned}
& \kappa=-3 \chi^{M} \chi_{M} \chi^{1}, \quad \kappa_{1}=-\chi^{M} \chi_{M}, \quad \kappa_{M}=-2 \chi^{1} \chi_{M}, \\
& \kappa_{11}=0, \quad \kappa_{1 M}=-\chi_{M}, \quad \kappa_{M N}=-\eta_{M N} \chi^{1}, \\
& \kappa_{a}=0, \quad \kappa_{\alpha a}=0, \quad \kappa_{a b}=\gamma_{M a b} \chi^{M} \\
& \left(\kappa^{-1}\right)^{11}=-\frac{3\left(\chi^{1}\right)^{2}}{\kappa}, \quad\left(\kappa^{-1}\right)^{1 M}=\frac{3 \chi^{M} \chi^{1}}{\kappa}, \\
& \left(\kappa^{-1}\right)^{M N}=-\frac{\eta^{M N}}{\chi^{1}}-\frac{3 \chi^{M} \chi^{N}}{\kappa}, \quad\left(\kappa^{-1}\right)^{a b}=\frac{\gamma_{M}^{a b} \chi^{M}}{\chi^{N} \chi_{N}} .
\end{aligned}
$$

Since we know from the previous sections that the Kähler submanifolds we get by doing the above truncation are again homogeneous, we can calculate the constant scalar curvature in one convenient point. Thus, we look at a point where $\lambda^{\mu}=0, \lambda^{2} \neq 0$, since the above expressions then simplify considerably. We get

$$
\bar{R}=-(q+2)^{2}-2(q+2) r-r^{2}-2+\frac{1}{2} \gamma_{M f a} \gamma_{b e}^{M} \delta^{a e} \delta^{b f}-\frac{1}{2} \gamma_{M f b} \gamma_{a e}^{M} \delta^{a e} \delta^{b f} .
$$

For the homogeneous non-symmetric space $L(0,1,1)$ we find $\bar{R}=-16$ for the dual space, while the original special Kähler space has $R=-18$, showing that generically the curvature tensors are different.

*Remember, we take $(\alpha)=(1, M)$ and $(a)=(i)$.

${ }^{\dagger}$ Here and in the appendix we used a shorthand notation, $\kappa_{. .} \equiv(\kappa \chi) . .,\left(\kappa^{-1}\right)^{*} \equiv\left((\kappa \chi)^{-1}\right) \cdot$. 
For the symmetric very special quaternionic manifolds, we also have $\gamma_{M(f b} \gamma_{a e)}^{M}=0$, so we finally get

$$
\bar{R}=-(q+2)^{2}-2-\frac{1}{2} q r-r^{2}-4 r .
$$

It is now easy to verify that we get the same value as for the original special Kähler manifolds that gave rise to the quaternionic manifolds via the c-map, just like in [9]. In fact, in the case $r=0$, since there are no $\gamma_{M}$-matrices, the only difference with the case studied in [8, 9] is the $S U(1,1)$ that was discarded. The truncated manifold is of the form $(S U(1,1) / U(1))_{1} \times(S U(1,1) / U(1))_{2} \times \mathcal{M}$, with $S U(1,1)_{1}$ generated by $\left\{\underline{\hat{\beta}}^{0}, \underline{\alpha}_{0}, \underline{\epsilon}_{0}-\frac{3}{2} \underline{\beta}\right\}$ and $S U(1,1)_{2}$ generated by $\left\{\underline{\hat{\alpha}}_{1}, \underline{\beta}^{1}, \underline{\epsilon}\right\}$. Taking $\mathcal{K}_{\text {dual }}=(S U(1,1) / U(1))_{2} \times \mathcal{M}$ corresponds to the case discussed in [8, 9 , while taking $\mathcal{K}_{\text {dual }}=(S U(1,1) / U(1))_{1} \times \mathcal{M}$ corresponds to the case discussed in this paper. Since both $S U(1,1) / U(1)$ parts have scalar curvature -2 , as is easy to verify, there is actually no difference between the two cases and so the dual Kähler spaces and the original very special Kähler spaces have, in the case of symmetric spaces, the same curvature tensor, like in 9 .

To study the full curvature tensor in the general case where $r \neq 0$, we make use of an extension of the computer program used in $[9]^{*}$. Let $z^{A}$ denote the coordinates of the original very special Kähler space, and $y^{A}=\Im z^{A}$. The metric can then be derived from the Kähler potential $K=-\log (V(y))$ and the curvature tensor is given by

$$
R_{A}{ }^{C}{ }_{B}{ }^{D}=-2 \delta_{(A}^{C} \delta_{B)}^{D}+\frac{4}{3} d_{A B E} C^{C D E}
$$

where $C^{A B C}$ is defined as in (2.10). In the symmetric case, it turns out that $C^{A B C}$ is constant [15], so that the curvature tensor $R_{A}{ }^{C}{ }_{B}{ }^{D}$ is constant (in this coordinate system). Similarly, we will denote the complex coordinates of the dual Kähler space as $\left(\eta^{A}\right)=$ $\left(\eta, \epsilon_{M}, \rho^{i}\right.$ ) (see previous notation) and the curvature tensor as $\bar{R}_{A}{ }_{B}{ }^{D}$. All components which are non-zero in the symmetric case are listed in the appendix and they can be derived from the Kähler potential $K=-2 \log V\left(\chi^{\alpha}\left(p\left(t_{\beta}, \lambda^{a}, \eta_{1}\right)\right)\right)-\log \left(\eta_{1}\right)$ (see section 3). Using the computer program mentioned before, we then find that the curvature tensor of the dual spaces are also constant in the symmetric case. To prove that the curvature tensors of the original special Kähler spaces and their dual spaces are the same, we need a (linear) coordinate transformation that relates the two tensors. This transformation can be found for all cases. We first transform the dual curvature tensor $\bar{R}$ to $\bar{R}^{\prime}$,

$$
\bar{R}_{A}^{\prime}{ }^{C}{ }_{B}{ }^{D}=U_{A}{ }^{A^{\prime}} U_{B}{ }^{B^{\prime}} R_{A^{\prime}}{ }^{C^{\prime}}{ }_{B^{\prime}}{ }^{\prime}\left(U^{-1}\right)_{C^{\prime}}{ }^{C}\left(U^{-1}\right)_{D^{\prime}}{ }^{D},
$$

with $U_{A}^{C}\left(U^{-1}\right)_{C}^{B}=\delta_{A}^{B}$ and $U_{A}^{B}=v^{A} \delta_{A}^{B}$ where $v^{1}=1, v^{2}=-2, v^{\mu}=2$ and $v^{i}=1$, and as before $\mu=3, \ldots, q+3$ runs over $q+1$ values, and $i=q+4, \ldots, n$ runs over $r$ values. We then find

$$
R_{A}{ }^{C}{ }_{B}{ }^{D}=\bar{R}_{C}^{\prime}{ }^{A}{ }^{B} \text {. }
$$

In the cases where $r=0$, the transformation $U$ has no effect and we find $R_{A}{ }^{C}{ }_{B}{ }^{D}=\bar{R}_{C}{ }^{A}{ }_{D}{ }^{B}$, as in 9, in agreement with our previous arguments.

\footnotetext{
*We would like to thank M. Trigiante for providing us with this program.
} 


\subsection{Flat potentials}

We will now show that the dual Kähler spaces give rise to flat potentials in $N=1$ supergravity. In order to establish this, we need to check the following equality [8]

$$
\frac{\partial K}{\partial \Re \eta^{A}}\left(\bar{g}^{-1}\right)^{A B} \frac{\partial K}{\partial \Re \eta^{B}}=3,
$$

where $\left(\eta^{A}\right)=\left(\eta, \epsilon_{M}, \rho^{i}\right)$. A straightforward calculation, using equations (3.9), (3.13) and

(5.11), shows that this is indeed the case. Notice that $\frac{\partial K}{\partial t_{M}}, \frac{\partial K}{\partial \lambda^{i}}$ and $\frac{\partial K}{\partial \eta_{1}}$ are independent of $t_{1}$ since $\bar{g}^{1 M}=\bar{g}_{i}{ }_{i}=\bar{g}_{0}{ }_{0}=0$. The calculations do not depend on the specific form of e.g $d_{\mu i j}$ and can therefore be generalized to non-homogeneous Kähler manifolds. Thus, we have found a new class of Kähler potentials that lead to flat potentials.

Notice that all the results in this section depend on the specific form (5.1) of the dsymbols and so do not apply to the case $S G_{5}$, characterized by e.g. $d_{111}=1$. In this case the original special Kähler space is $S U(1,1) / U(1)$ with scalar curvature $-2 / 3$ and $\mathcal{M}_{\text {trunc }}=(S U(1,1) / U(1))^{2}$, with one $S U(1,1) / U(1)$ also having scalar curvature $-2 / 3$ and the other having scalar curvature -2 . The $S U(1,1) / U(1)$ manifold with scalar curvature $-2 / 3$ gives rise to a flat potential, the other one does not.

\section{Conclusion}

In this paper, we studied the orientifold truncation that arises when compactifying type II string theory on Calabi-Yau orientifolds with O3/O7-planes. This truncation provides a way of reducing supersymmetry from $N=2$ to $N=1$, and we have studied the reduction of the hypermultiplet sector of $N=2$ supergravity with scalars spanning a $(4 n+4)$-dimensional very special quaternionic-Kähler manifold. We have established that the truncated $(2 n+2)$-dimensional manifold is Kähler, by explicitly determining the Kähler potential. Further, we studied the truncated isometry algebra. For homogeneous very special quaternionic-Kähler spaces, in the generic case, the truncated manifold splits into $S U(1,1) / U(1)$ times a $2 n$-dimensional Kähler space that can be viewed as dual to the original special Kähler space, in the sense of [8]. These spaces also give rise to flat potentials. For the case of symmetric spaces, the dual Kähler space consists of the same coset space as the original special Kähler space and has the same curvature tensor. We also studied a more general truncation for the symmetric spaces $L(0, P)$, and found that generically no $S U(1,1) / U(1)$ splits off anymore. Finally, one can also truncate symmetric special quaternionic-Kähler spaces that are not very special (this includes the case of minimal coupling): in this case the truncated manifold is still symmetric, but does not always factorize. The results are summarized in table (3).

\section{Acknowledgements}

We thank A. Van Proeyen for many helpful discussions, and for proofreading the manuscript. We have also greatly benefited from correspondence with S. Ferrara, R. D'Auria, 
M. Trigiante and T.W. Grimm. This work was supported in part by the European Community's Human Potential Programme under contract HPRN-CT-2000-00131 Quantum Spacetime and by the Belgian Federal Office for Scientific, Technical and Cultural Affairs through the Inter-University Attraction Pole P5/27.

\section{A Appendix: calculation of the curvature tensor.}

In this appendix we will give the components of the Christoffel connection and the curvature tensor which are necessary to calculate the scalar curvature of the truncated very special quaternionic-Kähler manifold. We also give all other components of the curvature tensor which are non-zero in the symmetric case. The connection and the Riemann tensor are defined as

$$
\begin{aligned}
\bar{\Gamma}_{\Sigma \Delta}^{\Lambda} & =\left(\bar{g}^{-1}\right)^{\Lambda \bar{\Lambda}} \partial_{\Sigma} \bar{g}_{\bar{\Lambda} \Delta}, \\
\bar{R}_{\Sigma}{ }^{\Gamma}{ }_{\Delta} \Lambda & =-\left(\bar{g}^{-1}\right)^{\Gamma \bar{\Gamma}} \partial_{\bar{\Gamma}} \bar{\Gamma}_{\Sigma \Delta}^{\Lambda} .
\end{aligned}
$$

Because the metric $\bar{g}_{\Lambda \bar{\Sigma}}$ is Kähler, the Christoffel connection is symmetric in the last two indices and the curvature tensor is symmetric under exchange of the first and the third index and/or the second and the fourth index.

After some long calculations, using equations (3.12) and (3.13) in section 3, we found the following results *

$$
\begin{aligned}
& \bar{\Gamma}_{\gamma}^{\alpha \beta}=\left(\bar{g}^{-1}\right)_{\gamma \bar{\gamma}} \frac{\partial \bar{g}^{\bar{\gamma} \beta}}{\partial t_{\alpha}}+\left(\bar{g}^{-1}\right)_{\gamma}{ }^{0} \frac{\partial \bar{g}_{0}{ }^{\beta}}{\partial t_{\alpha}}+\left(\bar{g}^{-1}\right)_{\gamma}{ }^{a} \frac{\partial \bar{g}_{a}{ }^{\beta}}{\partial t_{\alpha}} \\
& =d_{\bar{\alpha} \bar{\beta} \gamma}\left(\kappa^{-1}\right)^{\alpha \bar{\alpha}}\left(\kappa^{-1}\right)^{\beta \bar{\beta}}+\frac{6}{\kappa} \delta_{\gamma}^{(\alpha} \lambda^{\beta)}-\frac{3}{\kappa}\left(\kappa^{-1}\right)^{\alpha \beta} \kappa_{\gamma}, \\
& \bar{\Gamma}_{\gamma}^{\alpha}{ }^{\alpha}=\frac{1}{2 \eta_{1}^{2}}(\kappa \lambda \lambda)_{\beta} \bar{\Gamma}_{\gamma}^{\alpha \beta}+\frac{1}{\eta_{1}^{2}}(\kappa \lambda)_{a \bar{\gamma}}\left(\kappa^{-1}\right)^{\bar{\gamma} \alpha}\left(\kappa^{-1}\right)^{a c}(\kappa \lambda)_{\gamma c}-\frac{3}{\kappa \eta_{1}^{2}}(\kappa \lambda \lambda)_{\gamma} \lambda^{\alpha} \text {, } \\
& \bar{\Gamma}_{\gamma}^{\alpha a}=-\frac{1}{\eta_{1}}(\kappa \lambda \lambda)_{\beta a} \bar{\Gamma}_{\gamma}^{\alpha \beta}-\frac{1}{\eta_{1}} d_{a b \bar{\gamma}}\left(\kappa^{-1}\right)^{\bar{\gamma} \alpha}\left(\kappa^{-1}\right)^{b c}(\kappa \lambda)_{\gamma c}+\frac{3}{\kappa \eta_{1}}(\kappa \lambda)_{\gamma a} \lambda^{\alpha}, \\
& \bar{\Gamma}_{b}^{a \alpha}=-\left(\kappa^{-1}\right)^{a c} d_{b c \bar{\gamma}}\left(\kappa^{-1}\right)^{\bar{\gamma} \alpha}+\frac{3}{\kappa} \delta_{b}^{a} \lambda^{\alpha}, \quad \bar{\Gamma}_{0}^{0 \alpha}=0, \quad \bar{\Gamma}_{0}^{a \alpha}=-\bar{\Gamma}^{a \alpha}{ }_{b} \frac{\lambda^{b}}{\eta_{1}}, \\
& \bar{\Gamma}_{a 0}^{0}=\frac{1}{\eta_{1}} G_{a b} \lambda^{b}, \quad \bar{\Gamma}_{00}^{0}=-\frac{1}{\eta_{1}^{2}} G_{a b} \lambda^{a} \lambda^{b}-\frac{2}{\eta_{1}}, \quad \bar{\Gamma}_{a b}^{0}=-G_{a b}, \\
& \bar{\Gamma}_{b 0}^{a}=\frac{1}{2 \eta_{1}^{2}} d_{b c \bar{\gamma}}\left(\kappa^{-1}\right)^{\bar{\gamma} \alpha}(\kappa \lambda \lambda)_{\alpha}\left(\kappa^{-1}\right)^{a c}-\frac{1}{\eta_{1}^{2}}\left(\kappa^{-1}\right)^{a c}(\kappa \lambda)_{\bar{\gamma} c}(\kappa \lambda)_{\bar{\beta} b}\left(\kappa^{-1}\right)^{\bar{\gamma} \bar{\beta}} \\
& +\frac{3}{\kappa \eta_{1}^{2}}(\kappa \lambda \lambda)_{b} \lambda^{a}+\frac{3}{2 \kappa \eta_{1}^{2}}(\kappa \lambda \lambda)_{\alpha} \lambda^{\alpha} \delta_{b}^{a}-\frac{6 \lambda^{a}}{\kappa \eta_{1}^{2}} \kappa_{b c} \lambda^{c}-\frac{1}{\eta_{1}} \delta_{b}^{a}, \\
& \bar{\Gamma}_{b c}^{a}=\frac{2}{\eta_{1}} d_{\bar{\gamma} d(c}(\kappa \lambda)_{b) \alpha}\left(\kappa^{-1}\right)^{\bar{\gamma} \alpha}\left(\kappa^{-1}\right)^{a d}-\frac{6}{\kappa \eta_{1}} \delta_{(b}^{a}(\kappa \lambda \lambda)_{c)}+\frac{6 \lambda^{a}}{\kappa \eta_{1}} \kappa_{b c},
\end{aligned}
$$

${ }^{*}$ We used the following shorthand notation, $\kappa_{. .} \equiv(\kappa \chi) . .,\left(\kappa^{-1}\right) \cdot \equiv\left((\kappa \chi)^{-1}\right) \cdot$. 


$$
\begin{aligned}
& \bar{\Gamma}_{\alpha a b}=\frac{2}{\eta_{1}^{2}}\left(\kappa^{-1}\right)^{c e}\left(\kappa^{-1}\right)^{\beta \bar{\beta}}(\kappa \lambda)_{\alpha e} d_{\beta c(a}(\kappa \lambda)_{b) \bar{\beta}}-\frac{3}{\kappa \eta_{1}^{2}}\left(\kappa^{-1}\right)^{\beta \gamma}(\kappa \lambda)_{\beta a}(\kappa \lambda)_{\gamma b} \kappa_{\alpha} \\
& +\frac{d_{\bar{\beta} \bar{\gamma} \alpha}}{\eta_{1}^{2}}\left(\kappa^{-1}\right)^{\beta \bar{\beta}}\left(\kappa^{-1}\right)^{\gamma \bar{\gamma}}(\kappa \lambda)_{\beta a}(\kappa \lambda)_{\gamma b}-\frac{d_{\alpha a b}}{\eta 1}+\frac{3}{\kappa \eta_{1}^{2}}(\kappa \lambda \lambda)_{\alpha} \kappa_{a b}, \\
& \bar{R}_{\alpha}^{\beta}{ }^{\mu}{ }_{\gamma}=-\left(\bar{g}^{-1}\right)_{\alpha \bar{\alpha}} \frac{\partial}{\partial t_{\bar{\alpha}}} \bar{\Gamma}_{\gamma}^{\beta \mu}-\left(\bar{g}^{-1}\right)_{\alpha}{ }^{a} \frac{\partial}{\partial \lambda^{a}} \bar{\Gamma}_{\gamma}^{\beta \mu}-\left(\bar{g}^{-1}\right)_{\alpha}{ }^{0} \frac{\partial}{\partial \eta_{1}} \bar{\Gamma}_{\gamma}{ }^{\beta \mu} \\
& =-\delta_{(\alpha}^{\beta} \delta_{\gamma)}^{\mu}-d_{\bar{\beta} \bar{\mu}(\alpha} \kappa_{\gamma)}\left(\kappa^{-1}\right)^{\beta \bar{\beta}}\left(\kappa^{-1}\right)^{\mu \bar{\mu}}+\left(\kappa^{-1}\right)^{\beta \mu} \kappa_{\alpha \gamma} \\
& +\frac{\kappa}{3}\left(\kappa^{-1}\right)^{\bar{\beta}(\beta}\left(\kappa^{-1}\right)^{\mu) \bar{\mu}}\left(\kappa^{-1}\right)^{\delta \bar{\delta}} d_{\bar{\beta} \delta \alpha} d_{\bar{\mu} \bar{\delta} \gamma} \\
& \bar{R}_{c b}^{a b}=-\left(\bar{g}^{-1}\right)^{a}{ }_{\bar{\alpha}} \frac{\partial}{\partial t_{\bar{\alpha}}} \bar{\Gamma}_{b c}^{d}-\left(\bar{g}^{-1}\right)^{a e} \frac{\partial}{\partial \lambda^{e}} \bar{\Gamma}_{b c}^{d}-\left(\bar{g}^{-1}\right)^{a 0} \frac{\partial}{\partial \eta_{1}} \bar{\Gamma}_{b c}^{d} \\
& =-\delta_{(c}^{a} \delta_{b)}^{d}+\left(\kappa^{-1}\right)^{a d} \kappa_{c b}+\frac{\kappa}{3}\left(\kappa^{-1}\right)^{e(a}\left(\kappa^{-1}\right)^{d) f}\left(\kappa^{-1}\right)^{\gamma \bar{\gamma}} d_{\gamma e c} d_{\bar{\gamma} f b}, \\
& \bar{R}_{0}^{0{ }^{0}{ }^{0}}=-2, \quad \bar{R}_{\alpha 0}^{\beta}{ }^{0}=0, \quad \bar{R}_{b}{ }^{a}{ }^{0}=-\delta_{b}^{a}, \quad R_{a \gamma b}{ }^{0}=-d_{\gamma a b}, \\
& \bar{R}_{\alpha b}^{\beta}{ }^{a}=-\frac{1}{2} \delta_{\alpha}^{\beta} \delta_{b}^{a}+\frac{1}{2} d_{\bar{\beta} e b} \kappa_{\alpha}\left(\kappa^{-1}\right)^{\beta \bar{\beta}}\left(\kappa^{-1}\right)^{a e}-\frac{\kappa}{6}\left(\kappa^{-1}\right)^{a e}\left(\kappa^{-1}\right)^{c f}\left(\kappa^{-1}\right)^{\bar{\gamma} \beta} d_{\alpha c e} d_{\bar{\gamma} b f} \\
& -\frac{\kappa}{6}\left(\kappa^{-1}\right)^{a e}\left(\kappa^{-1}\right)^{\beta \gamma}\left(\kappa^{-1}\right)^{\bar{\gamma} \bar{\beta}} d_{\alpha \gamma \bar{\gamma}} d_{\bar{\beta} b e} \\
& \bar{R}_{0}^{\alpha a b}=\frac{\kappa}{6}\left(\kappa^{-1}\right)^{a e}\left(\kappa^{-1}\right)^{b c}\left(\kappa^{-1}\right)^{\gamma \alpha} d_{e c \gamma}-\frac{1}{2}\left(\kappa^{-1}\right)^{a b} \lambda^{\alpha} .
\end{aligned}
$$

All other components of the curvature tensor are zero in points of the manifold were $\lambda^{a}=0$ for all $a$. For the dual symmetric spaces this implies that these components are zero in every point.

\section{References}

[1] M. Grana, T. W. Grimm, H. Jockers and J. Louis, Soft supersymmetry breaking in Calabi-Yau orientifolds with D-branes and fluxes, Nucl. Phys. B690 (2004) 21, hep-th/0312232

[2] T. W. Grimm and J. Louis, The effective action of $N=1$ Calabi-Yau orientifolds, hep-th/0403067

[3] S. Ferrara and S. Sabharwal, Quaternionic manifolds for type II superstring vacua of Calabi-Yau spaces, Nucl. Phys. B332 (1990) 317

[4] J. Bagger and E. Witten, Matter couplings in N=2 supergravity, Nucl. Phys. B222 (1983) 1

[5] S. Hosono, A. Klemm and S. Theisen, Lectures on mirror symmetry, Nucl. Phys. B433 (1995) 501, hep-th/9403096 
[6] R. Böhm, H. Günther, C. Herrmann, and J. Louis Compactification of type IIB string theory on Calabi-Yau threefolds, Nucl. phys. B569 (2000) 229 hep-th/9908007

[7] B. de Wit, F. Vanderseypen, A. Van Proeyen, Symmetry structure of special geometries, Nucl. Phys. B400 (1993) 463, hep-th/9210068

[8] R. D'Auria, S. Ferrara and M. Trigiante, c-map, very special quaternionic qeometry and dual Kähler spaces, Phys. Lett. B587 (2004) 138, hep-th/0401161

[9] R. D'Auria, S. Ferrara and M. Trigiante, Homogeneous special manifolds, orientifolds and solvable coordinates, hep-th/0403204

[10] K. Becker, M. Becker, M. Haack, J. Louis, Supersymmetry breaking and $\alpha^{\prime}$-corrections to flux induced potentials, JHEP 0206 (2002) 060, hep-th/0204254

V. Balasubramanian and P. Berglund, Stringy corrections to Kähler potentials, SUSY breaking, and the cosmological constant problem, hep-th/0408054

[11] L. Andrianopoli, R. D'Auria and S. Ferrara, Supersymmetry reduction of $N$-extended supergravities in four dimensions, JHEP 0203 (2002) 025, hep-th/0110277

[12] L. Andrianopoli, R. D'Auria and S. Ferrara, Consistent reduction of $N=2 \rightarrow N$ $=1$ four dimensional supergravity coupled to matter, Nucl. Phys. B628 (2002) 387, hep-th/0112192

[13] E. Cremmer, S. Ferrara, C. Kounnas and D. V. Nanopoulos, Naturally vanishing cosmological constant in N=1 supergravity, Phys. Lett. B133 (1983) 61

[14] R. Barbieri, E. Cremmer and S. Ferrara, Flat and positive potentials in N=1 supergravity, Phys. Lett. B163 (1985) 143

[15] E. Cremmer, C. Kounnas, A. Van Proeyen, J. P. Derendinger, S. Ferrara, B. de Wit and L. Girardello, Vector multiplets coupled to $N=2$ supergravity: superhiggs effect, flat potentials and geometric structure, Nucl. Phys. B250 (1985) 385

[16] B. de Wit and A. Van Proeyen, Special geometry, cubic polynomials and homogeneous quaternionic spaces, Commun. Math. Phys. 149 (1992) 307, hep-th/9112027

[17] B. de Wit and A. Van Proeyen, Isometries of special manifolds, hep-th/9505097

[18] B. de Wit and A. Van Proeyen, Symmetries of dual quaternionic manifolds, Phys. Lett. B252 (1990) 221

[19] B. de Wit and A. Van Proeyen, Potentials and symmetries of general gauged $N=2$ supergravity-Yang Mills models, Nucl. Phys. B245 (1984) 89

[20] E. Cremmer and A. Van Proeyen, Classification of Kähler manifolds in $N=2$ vector multiplet-supergravity couplings, Class. Quant. Grav. 2 (1985) 445 